\title{
Energiekrise oder die Krise des Kapitalismus
}

\section{Massarrat}

\section{Vorbemerkung}

Die sogenannte Energiekrise hat wahrscheinlich die höchste Publizität unter allen anderen Krisenerscheinungen des Kapitalismus der letzten Jahre erreicht. Um so größer scheint aber auch die Verwirrung über die eigentlichen Ursachen dieser Krise zu sein. Die einen bringen die "Energiekrise ${ }^{66}$ mit dem Nahost-Konflikt in unmittelbaren Zusammenhang und die anderen erblicken hinter der Krise die gezielte Politik der USA-Regierung mit der Absicht, die Position des Dollars zu stärken.

Während die reaktionären Massenmedien auf die ,ölscheichs" verweisen und aktiv dabei sind, Rassenhaß zu schüren, indem sie die Misere des Kapitalismus anderen Völkern anzulasten versuchen, sehen Teile der Sozialisten die Ursache der „Energiekrise“ in der Existenz der multinationalen ölkonzerne begründet.

Hinter der gezielten Propaganda der einen und den Spekulationen und Illusionen der anderen bleiben notwendig die wirklichen Hintergründe verborgen.

Geht man aber, um die Besonderheiten der Wertproduktion in der Sphäre der Energieproduktion zu erfassen, von der Marxschen Analyse der kapitalistischen Wertproduktion aus, so stellt sich die sog. Energiekrise als eine besondere Krisenerscheinung der kapitalistischen Produktion heraus. Dies soll im folgenden Beitrag erklärt werden.

Hierzu ist zunächst erforderlich, die spezifische Wirkungsweise des Wertgesetzes in der extraktiven Sphäre, wozu auch die Sphäre der Energieproduktion gehört, kurz darzustellen. Diesem Schritt folgt dann, auf der Grundlage der modifizierten Wirkungsweise des Wertgesetzes, die Ableitung der Wertproduktion, des Tauschwertzusammenhangs verschiedener Energieträger, der Konkurrenz und der Bestimmungsmomente der Wert- bzw. Preisbildung auf dem Energiesektor.

Es ist klar, daß gerade im Zusammenhang mit der Produktion von Rohöl im Energiesektor der Frage der Aneignung des bei der Rohölproduktion erzielten Surplusprofits besondere Bedeutung zukommt. Deshalb soll dieser Frage und der Problematik des veränderten Verhältnisses des Imperialismus zu den rohstoffreichen Gesellschaften überhaupt und den hieraus folgenden Tendenzen besondere Aufmerksamkeit geschenkt werden.

\section{Die doppelte Modifikation des Wertgesetzes in der Sphäre der Rohstoffpro- duktion}

Im zehnten Kapitel des dritten Bandes des „Kapital“ leitet Marx die Wirkungsweise des Wertgesetzes unter der Voraussetzung seiner yollständigen Entfaltung ab, indem er von allen dem Kapital gegenüber fremden Kräften abstrahiert. Im sechsten Abschnitt desselben Buches, wo er die ,Verwandlung des Surplusprofits in Grundren- 
te" und damit seine Rententheorie ableitet, behandelt Marx in Wirklichkeit die modifizierte Wirkungsweise des Wertgesetzes in den unmittelbar von der Natur abhängigen Produktionssphären der Agrikultur und der Extraktion(1). In diesen Sphären wird das Wertgesetz in doppelter Weise modifiziert: zurm einen durch die Unmöglichkeit der Verallgemeinerung der höheren an die Naturkraft gebundenen Produktivkraft der Arbeit in der Sphäre, einen Umstand also, der die Konkurrenz einschränkt, und zum anderen durch das Grundeigentum -- in allen Fällen, wo Grund und Boden bzw. die Rohstoffquellen unter das Grundeigentum subsumiert sind -, das ebenfalls der Konkurrenz Schranken setzt. Im folgenden sollen diese beiden Formen der Modifikation des Wertgesetzes näher erläutert werden, da nur so die grundlegenden Kategorien zu einer umfassenden Behandlung der ökonomie der Rohstoffproduktion auf dem Energiesektor abgeleitet werden können.

\subsection{Die erste Modifikation des Wertgesetzes}

Die eigentlichen Ursachen der potenzierten Produktivkraft der Arbeit und die Möglichkeiten der Verallgemeinerung dieser Produktivkraft in einer Produktionssphäre sind an einer anderen Stelle ausführlich behandelt worden (2). Wir haben dort gesehen, daß die höhere Produktivkraft der Arbeit unter normalen Bedingungen aus dem Kapital selbst entspringt. Entweder dadurch, daß

„Kapital in größren als den durchschnittlichen Massen angewandt wird,“

oder aber dadurch, daß

„bessre Arbeitsmethoden, neue Erfindungen, verbesserte Maschinen, chemische Fabrikgeheim-

1) Die Bedeutung der Marxschen Rententheorie wurde bisher u. E. nur in beschränktem Umfang erkannt. Ihre Anwendung reduziert sich in der uns bekannten marxistischen Literatur auf die konkrete Erforschung des Kapitalverhältnisses zum Grundeigentum in der nationalen Agrikultursphäre. Die Bedeutung der Marxschen Rententheorie reicht aber weit über ihren bisherigen Anwendungsbereich hinaus. Sie ist der geeignetste theoretische Ansatz zur Erforschung des Verhältnisses des Kapitals zum Grundeigentum im internationalen Ausmaß und zur Ableitung der Modifikation des Wertgesetzes in allen unmittelbar von der Natur abhängigen Produktionssphären auf dem Weltmarkt. Der vorliegende Aufsatz liefert ein exemplarisches Beispiel für die Bedeutung der Marxschen Rententheorie, ohne deren Erkenntnis die Ableitung der wesentlichen Gründe für die sog. Energiekrise nicht möglich wäre. Die Marxsche Rententheorie ist $u$. E. auch die fundierteste theoretische Grundlage der klassischen Weltarbeitsteilung.

Im Wintersemester 1973/74 wurde die Marxsche Rententheorie im Rahmen einer Übung am Otto-Suht-Institut der Freien Universität Berlin eingehend behandelt. Im Sommersemester 1974 soll diese Übung, in deren Mittelpunkt die konkrete Anwendung der Rententheorie steht, fortgesetzt werden.

2) Massarrat, M.: Zur Problematik der gleichen Mehrwertrate und Kritik der Marxschen Theorie der absoluten Grundrente. Dieser Aufsatz, in dem sich der Verfsser mit der Annahme der gleichen Mehrwertrate und mit der Marxschen Theorie der absoluten Grundrente kritisch auseinandersetzt, wurde zwar im Oktober 1973 verfaßt, soll jedoch zunächst intern diskutiert und erst zu einem späteren Zeitpunkt veröffentlicht werden. 
nisse etc., kurz neue, verbesserte, über dem Durchschnittsniveau stehende Produktionsmittel und Produktionsmethoden ange wandt werden" (3).

Es wird dort ferner abgeleitet, daß diese höhere Produktivkraft der Arbeit in der Sphäre verallgemeinert, also in die durchschnittliche Produktivkraft der Arbeit, die in der Sphäre angewandt wird, verwandelt werden kann. Der Marktpreis bzw. der allgemeine Produktionspreis (Kostpreis plus Durchschnittsprofit) der Waren in der Sphäre wird durch den individuellen Produktionspreis der Waren jener Kapitale reguliert, die den bedeutenden Anteil der Waren in der Sphäre produzieren. Kapitale, die unter besseren Bedingungen produzieren, also eine Produktivkraft anwenden, die höher ist als die mittlere Produktivkraft der Arbeit, realisieren einen Surplusprofit, da der individuelle Produktionspreis ihrer Waren unter dem allgemeinen Produktionspreis derselben liegt; und Kapitale, die unter den Bedingungen produzieren, die ungünstiger sind als die mittleren Bedingungen derselben Sphäre, sind nicht in der Lage, den Durchschnittsprofit zu realisieren, da der individuelle Produktionspreis ihrer Waren über dem Marktpreis derseiben liegt, weshalb sie auch über kurz oder lang die Produktion einstellen müssen.

Wenn aber beim Gleichgewichtszustand der Nachfrage und der Zufuhr oder aber auch bei kurzfristiger Abweichung der Nachfrage von der Zufuhr nicht die unter den schlechtesten Bedingungen produzierenden Kapitale in einer Produktionssphäre den Marktpreis regulieren, sondern jene, die unter den mittleren Bedingungen produzieren, so deshalb, weil unter Konkurrenzbedingungen die Kapitale, die unter mittleren bzw. unter günstigsten Bedingungen der Sphäre produzieren, durch die Ausweitung ihres Marktanteils das anstehende gesellschaftliche Bedürfnis nach Waren dieser Sphäre kurzfristig und vollständig decken können, die unter den schlechtesten Bedingungen produzierenden, die gesellschaftliche Arbeit vergeudenden Kapitale also ersetzen können.

Anders als unter den Konkurrenzbedingungen, z.B. in der Industriesphäre, kann in den unmittelbar von der Natur abhängigen Produktionssphären weder die höhere Produktivkraft der Arbeit verallgemeinert werden, noch wird daher der Marktpreis durch den individuellen Produktionspreis der unter den ,mittleren Bedingungen " produzierten Waren reguliert.

In der extraktiven Sphäre (wie in der Agrikultur, die wir aber der Einfachheit halber in unserer Betrachtung ausnehmen) ist đie stoffliche Basis der höheren Produktivkraft der Arbeit die Naturkraft, eine von der Natur vorgegebene Produktionsbedingung also, die bestimmten Kapitalen eine höhere Verwertung dieser von ihnen angewandten Produktivkraft der Arbeit erlaubt, vorausgesetzt, daß alle in der Sphäre fungierenden Kapitale die gleiche mittlere organische $\mathbb{Z}$ usammensetzung haben. Die höhere Produktivkraft der Arbeit in der extraktiven Sphäre entspringt also nicht aus dem Kapital, sondern

„, sie entspringt aus der größren naturwüchsigen Produktivkraft der Arbeit, gebunden an die Benutzung einer Naturkraft, aber nicht einer Naturkraft, die allem Kapital in derselben Produktionssphäre zur Verfügung steht, (. . ) sondern einer monopolisierten Naturkraft, die wie der

3) Marx, Karl: Das Kapital. Kritik der politischen Ökonomie. Berlin 1969. Bd. III, S. 657 
Wasserfall nur denen zur Verfügung steht, die über besondre Stïcke des Erdbodens und seine A ppartenentien $z u$ verfugen haben ${ }^{66}(4)$.

Kapitale, die eine an die ausnahmsweise günstige Naturkraft gebundene Produktivkraft der Arbeit anwenden, erzielen einen Surplusprofit, der sich von der Verwertungsseite her von dem gewöhnlichen Surplusprofit durch nichts unterscheidet. Indes ist die ausnahmsweise günstige Naturkraft selbst keineswegs die Quelle des Surplusprofits:

„Die Naturkraft ist nicht die Quelle des Surplusprofits, sondern nur eine Naturbasis desselben, weil die Naturbasis der ausnahmsweise erhöhten Produktivkraft der Arbeit. So ist der Gebrauchswert ïberhaupt Träger des Tauschwerts, aber nicht seine Ursache" (5).

Der wesentliche Unterschied der extraktiven Sphäre zur Industriesphäre ist also die Tatsache, daß in der extraktiven Sphäre die durch die Natur bedingte höhere Produktivkraft der Arbeit nicht verallgemeinert werden kann, weil die Bestimmtheit ihrer stofflichen Basis eine vom Kapital unabhängige, von der Natur vorgegebene ist. Die erste Modifikation des Wertgesetzes in dieser Sphäre findet also ihren Ausdruck darin, daß der Konkurrenz der Einzelkapitale Schranken gesetzt sind, die Produktivkraft der Arbeit zu steigern und diese vermittels Ausdehnung ihres Marktanteils in der Sphäre zu verallgemeinern, den regulierenden Marktpreis also tendenziell zu senken. Die Konkurrenz der Einzelkapitale besteht in der Sphäre in der Hauptsache nur noch darin, das Monopol an der besonders günstigen, von der Natur vorgegebenen stofflichen Basis der Produktivkraft zu erwerben, um sich einen dauerhaften Surplusprofit zu sichern, um die eigentliche Konkurrenz also auszuschalten.

Da auf der einen Seite die Naturbasis der Produktivkraft - sei nun diese Naturbasis Grund und Boden oder aber sei sie mineralischer Rohstoff - nicht beliebig reproduziert werden kann, hängt der Marktanteil der Einzelkapitale an den Waren dieser Sphäre davon ab, über welches Monopol von Quantität und Qualität der natürlichen Basis der Waren dieser Sphäre die Einzelkapitale verfügen. Auf der anderen Seite ist es ein außergewöhnlicher Zufall, daß die denkbar günstigste von der Natur vorgegebene stoffliche Bedingung der Produktion eines Rohstoffs, ob an einem einzigen Ort oder an mehreren Teilen des Erdballs verteilt, in uneingeschränktem Umfang vorkommt. In der Regel kommt die stoffliche Basis der Rohstoffproduktion in relativ beschränkter Quantität vor und ist mit gravierendem Unterschied in der Qualität sowie im Standort auf unseren Erdball verteilt. Die höhere bzw. mindere stoffliche Qualität eines Rohstoffes, die sich letztlich auf dem Markt ökonomisch niederschlägt, ergibt sich aus der chemischen Zusammensetzung des Rohstoffes, den natürlichen Verhältnissen seiner Produktion, seiner Transportierbarkeit und den Bedingungen seiner stofflichen Verarbeitung. Es darf als erwiesen gelten, daß z. B. Rohöl gegenüber der Steinkohle als Energieträger in allen oben aufgezählten Punkten erhebliche Vorteile aufweist.

Diese von der Natur vorgegebenen stofflichen Bedingungen der Rohstoffproduktion bedeuten für die Kapitalverwertung, daß der individuelle Produktionspreis

4) Ebd., S. 658

5) Ebd., S. 659 f. Hervorh. d. Verf. 
der von Einzelkapitalen produzierten Rohstoffe einer Sphäre, z. B. des Energiesektors, je nachdem, ob sie über das Monopol von minderer oder besserer Qualität der Naturbasis des Rohstoffs verfügen, unterschiedlich ist. An der Tatsache, daß der individuelle Produktionspreis der Rohstoffe einer Sphäre von verschiedenen Quellen mit unterschiedlicher Qualität und unterschiedlichem Standort verschieden ist, ändert sich auch nichts, wenn das Monopol der gesamten verfügbaren Quellen dieses Rohstoffes einem einzigen Kapital unterworfen ist. Die Frage ist aber hier, wodurch eigentlich der Marktpreis der Rohstoffe dieser Sphäre reguliert wird.

Zur vollständigen Befriedigung des gesellschaftlichen Bedürfnisses - im nationalen wie im internationalen Sinne - nach einem bestimmten Rohstoff müssen der Voraussetzung nach Rohstoffe derselben Art aber mit verschiedenen individuellen Produktionspreisen produziert werden. Wir haben gesehen, daß in der Industrie Kapitale mit ungünstigsten Produktionsbedingungen gesellschaftliche Arbeit vergeuden und daher von den Kapitalen mittlerer oder günstigster Produktionsbedingungen vom Markt verdrängt werden können, weil der Ausdehnung des Marktanteils der letzteren keine äußerlichen Schranken gesetzt sind. Wir haben aber auch gesehen, daß in der extraktiven Sphäre die angewandte höhere Produktivkraft der Arbeit, weil ihre Naturbasis, nicht verallgemeinert werden kann.

Unter diesen Bedingungen verwertet sich in der extraktiven Sphäre auch die vom Kapital angewandte niedrigste Produktivkraft der Arbeit als die gesellschaftlich notwendige Arbeit. Dies allerdings nur dann, wenn die vollständige Befriedigung des gesellschaftlichen Bedürfnisses auch die Produktion von Rohstoffen der qualitativ oder standortmäßig ungïnstigsten Naturbasis, an die die Produktivkraft der Arbeit gebunden ist, erforderlich macht. Das bedeutet aber, daß auch das Kapital, das die niedrigste Produktivkraft der Arbeit anwendet, den normalen, den durchschnittlichen Profit erzielen, daß also der individuelle Produktionspre is seiner Ware, zumindest dem Marktpreis entsprechen muß. Daher wird der Marktpreis in der extraktiven Sphäre im Unterschied zur Industriesphäre, durch den individuellen Produktionspreis der unter den schlechten Bedingungen produzierten Waren reguliert.

Steigt nämlich das gesellschaftliche Bedürfnis nach Produkten der Sphäre an, so daß die Produktion von qualitativ oder standortmäßig noch ungünstigeren Rohstoffen unvermeidlich wird, deren individueller Produktionspreis höher ist als der bisher den Marktpreis regulierende individuelle Produktionspreis, so muß der Marktpreis soweit ansteigen, bis die durchschnittliche Verwertung des Kapitals auch bei der Produktion von Rohstoffen unter den ungünstigsten Verhältnissen möglich ist.

Sinkt dagegen das gesellschaftliche Bedürfnis nach dem Rohstoff oder werden neue qualitativ bzw. standortmäßig günstigere Quellen dieser Rohstoffe entdeckt, so kann das Kapital, das bisher unter den ungünstigsten Bedingungen produzierte, dessen Waren also den höchsten den Markt regulierenden individuellen Produktionspreis hatten, den Durchschnittsprofit nicht mehr realisieren, da unterdiesen Bedingungen die Konkurrenz der unter besseren Bedingungen produzierenden Kapitale den Marktpreis herabdrückt, und zwar auf den individuellen Produktionspre is jener Rohstoffgruppe in der Sphäre, deren Produktion das gesellschaftliche Bedürfnis gerade noch deckt. Die vom Kapital bisher unter den schlechtesten Bedingungen angewandte Produktivkraft der Arbeit erweist sich unter den veränderten Bedingun* 
gen als gesellschaftlich vergeudete Arbeit, weshalb auch dieses Kapital vom Markt ausgeschaltet wird.

Unter allen diesen Bedingungen wird der Marktpreis der Rohstoffe auf jeden Fall durch den individuellen Produktionspreis der unter den ungünstigen Bedingungen produzierten Rohstoffe derselben Art reguliert. Kapitale, die diese Gruppe von Rohstoffen produzieren, erzielen lediglich den Durchschnittsprofit. Die übrigen Kapitale, die unter günstigen Bedingungen in der Sphäre produzieren, deren individueller Produktionspreis also niedriger ist als der Marktpreis, erzielen je nachdem wie günstig Qualität und Standort der Naturbasis des Rohstoffes sind, über deren Monopol sie verfügen, einen geringeren oder höheren Surplusprofit. Und diese Surplusprofite fallen an, unabhängig davon, ob die Rohstoffquellen unter das $G$ rundeigentum subsumiert sind oder nicht. Das Grundeigentum kann höchstens bewirken, daß der Surplusprofit in Grundrente verwandelt wird, ein Umstand, der aber vom historischen Verhältnis des Kapitals zum Grundeigentum abhängt. Darauf gehen wir später noch ein.

\subsection{Die zweite Modifikation des Wertgesetzes}

Bei der obigen Analyse der ersten Modifikation des Wertgesetzes im extraktiven Sektor haben wir unterstellt, daß der Konkurrenz der Kapitale in der Sphäre keine Schranken gesetzt sind, neue qualitativ bzw. standortmäßig günstigere Rohstoffquellen zu erwerben, ihren Konkurrenzkampf also durch permanente Zuflucht zu Rohstoffquellen höherer Qualität auszutragen. Der Konkurrenz waren, wie oben beschrieben, nur insofern Schranken gesetzt, als Kapitale, die die höhere Produktivkraft anwenden, diese in der Sphäre nicht verallgemeinern können. Hier lassen wir diese Annahme fallen und gehen davon aus, daß die Naturbasis der Rohstoffe insgesamt unter das Grundeigentum subsumiert ist, daß also das Grundeigentum, als ein dem Kapital fremdes Moment, das Kapital an der uneingeschränkten Wahl der Naturbasis seiner Produktion hindert, daher der Konkurrenz der Kapitale untereinander urm die Steigerung der Produktivkraft der Arbeit durch die Anwendung der günstigeren Naturbasis Schranken setzt.

Das Grundeigentum schließt die besonders günstige Naturbasis der Rohstoffe solange von der Produktion aus, bis ihm für die Erlaubnis, diese günstige Naturbasis für die Rohstoffproduktion anzuwenden, eine Gebühr, eine Steuer bezahlt wird. Die Konkurrenz der Kapitale um das Monopol an der günstigeren Naturbasis befähigt das Grundeigentum, solange die Gebühr hochzuschrauben, wie die Kapitale den gesamten Surplusprofit an das Grundeigentum abgeben und nur noch gerade den normalen Durchschnittsprofit erzielen können.

Unter diesen Bedingungen fehlt dem Kapital jeder Anreiz, die Produktivkraft der Arbeit durch die Anwerdung von qualitativ bzw. standortmäßig günstigerer Naturbasis der Rohstoffe zu steigern, da der zu realisierende Extraprofit sowieso vom Grundeigentum abgezweigt wird. Das Grundeigentum wirkt also hier insofern als Schranke des Kapitals, als es verhindert, die der Konkurrenz entgegenwirkenden Momente dadurch aufzuheben, daß eine immer vorteilhaftere Naturbasis der Pro- 
duktion angewendet wird.

Das Grundeigentum modifiziert das Wertgesetz in der extraktiven Sphäre also noch einmal, um das Kapital daran zu hindern, die erste Modifikation desselben in der Sphäre aufzuheben.

\subsection{Die Verwandlung des Surplusprofits in Grundrente}

Ist die gesamte Naturbasis der Rohstoffe unter das Grundeigentum subsumiert, so wird der Surplusprofit in Grundrente verwandelt. Marx unterscheidet dem Wesen nach zwei verschiedene Formen der Grundrente: die Differentialrente und die absolute Grundrente.

Bei dem oben angeführten Fall des Surplusprofits, der aus der Differenz des individuellen Produktionspreises der Einzelkapitale und des allgemeinen Produktionspreises in der Sphäre entspringt, handelt es sich um die Differentialrente, wenn dieser Surplusprofit vom Grundeigentum angeeignet wird.

„Es ist klar, daß diese Rente immer Differentialrente ist, denn sie geht nicht bestimmend ein in den allgemeinen Produktionspreis der Ware, sondern setzt ihn voraus. Sie entspringt stets aus der Differenz zwischen dem individuellen Produktionspreis des Einzelkapitals, dem die monopolisierte Naturkraft zur Verfügung steht, und dem allgemeinen Produktionspreis des in der fraglichen Produktionssphäre überhaupt angelegten Kapitals“ (6).

Die absolute Grundrente wird dagegen bestimmt vom Grundeigentum jener in jeder Hinsicht ungünstigsten, vom Kapital aber angewendeten, Naturbasis des Rohstoffes. Das Kapital wendet diese vergleichsweise fragliche ungünstigste Naturbasis noch an, weil es sich aufgrund des gegebenen Verhältnisses der Nachfrage zur Zufuhr gerade noch durchschnittlich verwerten kann. Aber diese durchschnittliche Verwertung des Kapitals ist kein Anlaß für das Grundeigentum, die diesem unterworfene Naturbasis, so schlecht sie auch sein mag, dem Kapital umsonst zur Verfügung zu stellen. Das Grundeigentum schließt diese ihm gehörige ungünstigste Naturbasis solange von der Produktion aus, bis ihm eine Gebühr gezahlt werden kann, bis also das gesellschaftliche Bedürfnis nach dem Rohstoff dieser Naturbasis die Zufuhr langfristig übersteigt, folglich der Marktwert, und damit der Marktpreis, über den allgemeinen Produktionspreis des in der Sphäre überhaupt angelegten Kapitals ansteigt. Diese Differenz des Marktpreises der fraglichen Ware von dem allgemeinen Produktionspreis derselben, als eine besondere Form des Surplusprofits, des natürlichen Monopolprofits, der in den Preis der Ware eingeht, verwandelt sich in Grundrente, in absolute Grundrente, indem sie vom Grundeigentum angeeignet wird.

Wie wir in der Auseinandersetzung mit der Marxschen Theorie der absoluten Grundrente gesehen haben (7), kann sich aber das Grundeigentum in der kapitalistischen Gesellschaft nur dann als solches verwirklichen, als Schranke des Kapitals also fungieren, wenn die unter das Grundeigentum subsumierte stoffliche Basis einer Ware nach der ein zahlungsfähiges gesellschaftliches Bedürfnis existiert, in relativ be-

6) Ebd., S. 659

7) Vgl. dazu Massarrat, M.: Zur Problematik der gleichen Mehrwertrate ..., 
schränktem Umfang vorhanden ist.

Ist dagegen die Naturbasis der fraglichen Ware in der extraktiven Sphäre, in der Agrikultur etc. unbeschränkt vorhanden, wie z. B. das Meerwasser als Naturbasis von Fischen, dann führt die Konkurrenz der Grundeigentümer untereinander - falls diese Naturbasis überhaupt unter das Grundeigentum subsumiert wird - zur tenden. ziellen Senkung der Grundrente (sowohl die Differentiolrente wie die absolute Grundrente) und schließlich zur voliständigen Aufhebung des Grundeigentums als Schranke des Kapitals.

\section{Das Wertgesetz und der Energiesektor}

Die oben aufgezeigten theoretischen Grundvoraussetzungern gestatten uns nun, der Behandlung des eigentlichen Problems, nämlich der Analyse der Besonderheit der Wert- und Preisbildung, der Konkurrenz etc. auf dem Energiesektor, einen Schritt näherzukommen. Es ist klar, daß der Energiesektor nicht auf nationaler Ebene, sondern international gesehen werden muß, da der Marktpreis für die Produkte diese Sphäre, wie für die Produkte aller von der Natur unmittelbar abhängigen Produktionssphären, im internationalen Zusammenhang überhaupt ableitbar ist. Der Einfachheit halber abstrahieren wir von der Modifikation des Wertgesetzes durch die Währungsmechanismen und beschränken uns auf die Wirkung der sich unmittelbar im Produktionsprozeß durchsetzenden beiden oben aufgezeigten Modifikationen des Wertgesetzes, da die erstgenannte Form eine selbständige, also getrennt zu behandelnde Modifikationsform desselben ist.

Die kohlenstoffhaltigen Energieträger Rohöl, Steinkohle, Braunkohle, Erdgas, Ölschiefer etc., so wie sie als Gebrauchswertform in der Natur vorkommen, unterscheiden sich in ihrer Form, in ihrer chemischen Zusammensetzung und einer Reihe physikalisch-chemischer Eigenschaften grundlegend. Ihre einzige gebrauchswertmäBige Gemeinsamkeit besteht darin, daß sie alle kohlenstoffhaltig sind. Nehmen wir die wichtigsten nicht kohlenstoffhaltigen Energieträger, wie die Kernenergie, thermodynamische Energiequellen, Stauseen etc. noch hinzu, dann ist in allen Formen der Enrgieträger nicht einmal eine stoffliche Gemeinsamkeit feststellbar. Die allgemeine Bestimmung dieser Energieträger als Ware auf dem Markt ist, daß sie, wie jede Ware überhaupt, einen Tauschwert besitzen. Thre besondere Bestimmung und ihre zweite Gemeinsamkeit als Ware besteht aber darin, daß sie alle miteinander in einem Tauschwertzusammenhang stehen, weil sie alle die verschiedenen Gebrauchswertformen einer und derselben Ware sind.

Die Energieträger haben - von den Nebenprodukten zunächst abstrahiert ausschließlich deshalb einen Tauschwert, weil sie alle die stofflichen Träger einer und derselben Warenform, der Energie also, sind, die sich unter den verschiedensters technischen Stoffwechselprozessen schließlich in Wärme verwandeln. Der Wert, daher also der Marktpreis, einer bestimmten Menge der oben aufgeführten Energieträger ergibt sich also daraus, wieviel Energie, in Kalorien ausgedriickt, diese schließlich abgeben und welche Kosten für die Trennung der Wärmeenergie von ihrem stofflichen Träger jeweils anfallen. 
Um nun die Wirkungsweise des Wertgesetzes auf dem internationalen Energiesektor anschaulich darzustellen, lassen wir alle jene Formen der Energieträger unberücksichtigt, denen wegen ihres relativ geringen Anteils an der Energieversorgung der Welt insgesamt keine Bedeutung zukommt und die daher auf die Wert- und Preisbestimmung der Energieträger keinen Einfluß haben. Hierzu gehören alle nicht kohlenstoffhaltigen Energieträger sowie Ölschiefer, Erdgas und Braunkohle. Zurück bleiben also, wie aus Abbildung 1 (7a) hervorgeht, die beiden gegenwärtig als Hauptenergieträger der Welt geltenden Rohstoffe Steinkohle und Rohöl.

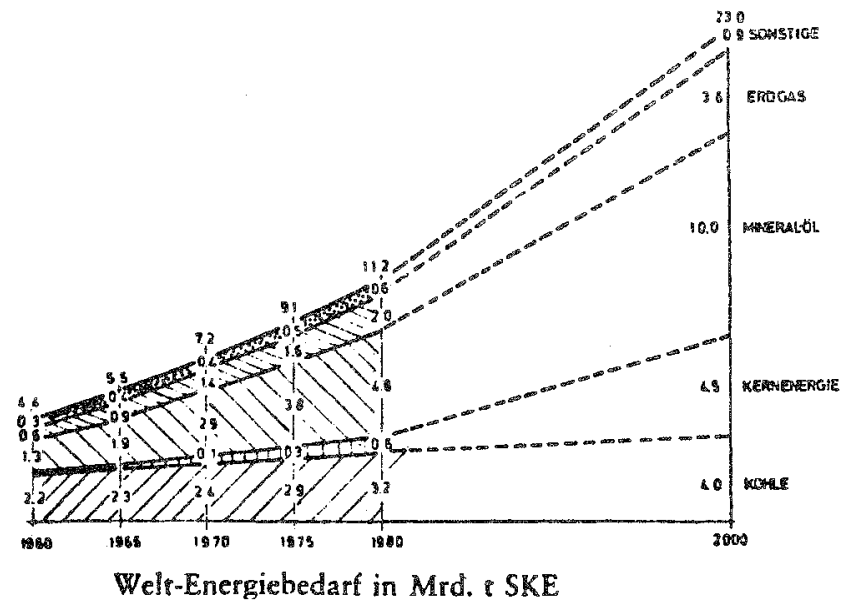

Die Annahme dürfte unzweifelhaft zutreffen, daß Rohöl als besondere Gebrauchswertform der Energie gegenüber der Steinkohle als einer anderen Gebrauchswertform derselben in allen Bereichen, von seiner unmittelbaren Produktion bis zu seiner unmittelbaren Verwandlung in Wärmeenergie, erhebliche Vorteile aufweist. Hinzu kommt die Tatsache, daß die Nebenprodukte des Rohöls in allen Industriesektoren zu vielfältigen $Z$ wecken Verwendung finden, daß aber die Beseitigung von Rückständen der Steinkohle zusätzliche Kosten verursacht.

Wenn wir selbst von allen Vorteilen des Rohöls gegenüber der Steinkohle bei der Verarbeitung und von allen sich ökonomisch hoch verwertenden ölnebenprodukten absehen und lediglich die Menge von Kalorien, die Rohöl bzw. Steinkohle beinhaltet als die Hauptbestimmung des Marktwertes bzw. des Marktpreises beider Gebrauchswertformen der Energieträger zugrundelegen, so müssen wir feststellen, dafs der individuelle Produktionspreis (Kostpreis, worin Transportkosten eingeschlossen + Durchschnittsprofit) einer Tonne Steinkohle, auf die darin enthaltene Menge Kalorien umgerechnet, ein vielfaches vom individuellen Produktionspreis einer Tonne Rohöl darstellt, da zur Produktion von einer bestimmten Menge Energie in Form von Steinkohle aufgrund ihrer besonders ungünstigen Gebrauchswert form die mehrfache Kapitalmasse notwendig ist als es zur Produktion von derselben Kalorienmenge in Form von Rohöl erforderlich wäre.

7a) Quelle: Erdol und Kohle, Hermhausen 1973, Dez. 1973, S. 715 a. a. O. (vgl. Fußnote 2) 
Die Produktivkraft der Arbeit bei der Produktion von Rohöl ist also mehrfach höher als jene bei der Produktion von Steinkohle, da die stoffliche Basis der ersteren eine günstigere ist als jene der letzteren. $\mathrm{Da}$ die stoffliche Basis der Produktivkraft der Arbeit in der Sphäre eine von der Natur vorgegebene ist, die also nicht verallgemeinert werden kann, sind dieselben Gesetze auf dem Energiesektor wirksam, die oben unter 2. abgeleitet wurden. Demnach reguliert der individuelle Pro. duktionspreis der Steinkohle den Marktpreis für alle anderen zumindest kohlenstoffhaltigen Gebrauchswertformen der Energieträger, weil erstens dieser der höchste in der Sphäre ist und weil zweitens und dennoch nach Steinkohle auf dem internationalen Energiemarkt ein großes zahlungsfähiges Bedürfnis vorhanden ist.

Daß auch in der Tat die Steinkohle den Marktpreis aller anderen Formen des Energieträger bestimmt, kommt u. E. darin zum Ausdruck, daß der mittlere Energiegehalt der Steinkohle sich als die Umrechnungsbasis für alle anderen Formen der Energieträger durchgesetzt hat ( 8 ).

Das folgende Schema (9) zeigt deutlich, daf der individuelle Produktionspreis - im Schema Förderkosten (10) - des Rohöls z. B. am Persischen Golf im Ok tober 1973 weniger als $2 \%$ des Marktpreises ausmachte und daß heute bei einem Preis von durchschnittlich 10 Dollar pro Barrel Rohöl dieser lediglich $1 \%$ des Marktpreises ausmacht.

Aus diesem Schema geht übrigens auch deutlich hervor, daß der Marktwert und der Marktpreis des Rohöls am Persischen Golf mit seinem individuellen Produktionso preis nicht das geringste zu tun hat, der Marktpreis des Rohöls also durch den individuellen Produktionspreis anderer Arten von Energieträgern reguliert werden muß.

Indes sind die individuellen Produktionspreise der Steinkohle verschiedener Regionen durchaus unterschiedlich, genau so wie die individuellen Preise des Rohöls

8) Der Begriff Steinkohleneinheit (SKE) entspricht dem mittleren Energiegehalt von $1 \mathrm{~kg}$ Steinkohle $=7000 \mathrm{kcal}$. Die SKE wird in der Energiewirtschaft vielfach als bloße technisch statistische Größe bezeichnet, dürfte aber. in Wirklichkeit eher ökonomisch begründet sein. Der Energiegehalt von verschiedenen Energieträgern auf $\mathrm{kg}$ umgerechnet, beträgt demnach für Erdölgas 1,471, Rohöl 1,429, Erdgas 1,286, Steinkohle 1,0 und für Braunkohle 0,286 SKE.

9) Quelle: Die Zeit vom 13. 12. 1973. Der Gewinnanteil der Förderländer am Surplusprofit beträgt zwar formal $55 \%$. Dadurch aber, daß bisher nicht der Marktpreis am Bestimmungsort, sondern der sog. „Posted Price“, also der Rohölpreis am Golf von Mexiko minus Transportkosten vom Persischen Golf bis zum Golf von Mexiko und unter Abzug von US-Zollkosten, also Kosten, die in Wirklichkeit gar nicht entstanden, ermittelt wurde, ergab sich für die Förderländer real ein wesentlich niedrigerer Rohölpreis und daher Gewinnanteil der weiter unter $55 \%$ des aus dem wirklichen Marktpreis errechneten Gewinnanteils lag. Vgl. dazu: Frankfurter Rundschau vom 31. 10.1973, \$. 3. Wie manipuliert die wirklichen Relationen in der bürgerlichen Presse wiedergegeben werden, geht auch aus dem Vergleich des obigen Schemas mit der graphischen Darstellung der $\mathrm{Abb} .4$ hervor.

10) Aus dem Begriff "Förderkosten" geht nicht hervor, ob es sich dabei um den Kostpreis oder aber um den Produktionspre is handelt. Selbst wenn in „F örderkosten" der Durchschnittsprofit nicht berïcksichtigt ist, dürfte sich das Ergebnis, angesichts der kaum ins Gewicht fallenden, im Bereich der Rohölproduktion insgesamt angelegten Kapitalmasse, auf die sich der Durchschnittsprofit bezieht, nicht wesentlich ändern. 


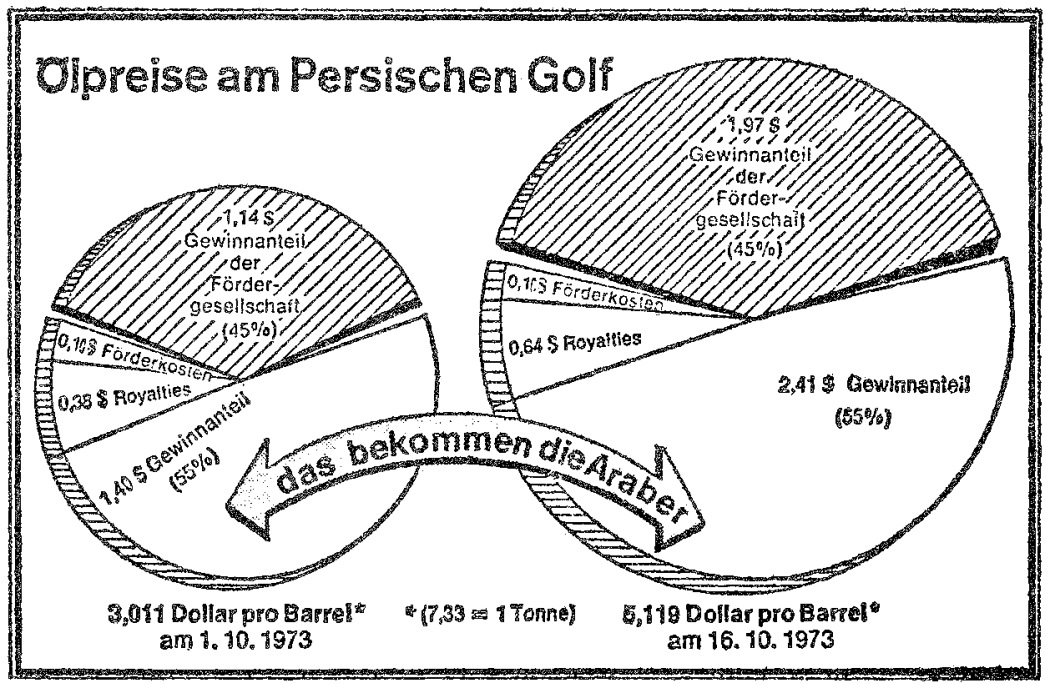

verschiedener Gebiete gravierende Unterschiede aufweisen. Der individuelle Produktionspreis des Rohöls am Persischen Golf und in Afrika ist erheblich niedriger als jener des amerikanischen Rohöls, weil nicht nur die Qualität der ersteren besser ist, im Durchschnitt weniger schädliche Beimengungen wie Schwefel etc. und mehr SKE enthält, die Produktionskosten auf SKE umgerechnet, also niedriger sind, sondern weil aufgrund der von der Natur vorgegebenen günstigen Bedingungen die Förderung des Rohöls am Persischen Golf und in Afrika bis zur Erdoberfläche mehr oder weniger auf natürliche Weise erfolgt, die Förderleistung je Sonde daher bis zu tausendfach höher als jene in Amerika ist (11).

Der individuelle Produktionspreis der amerikanischen Steinkohle ist wiederum niedriger als der der europäischen Steinkohle. Auch hier ist der Unterschied der Produktionspreise zunächst auf die Unterschiede der natürlichen Bedingungen der Produktion zurückzuführen. Die Beimengungen der amerikanischen Steinkohle, z. B. der Aschegehalt, sind geringer, der Wärmegehalt daher höher als der der europäischen Steinkohle, so daß der individuelle Produktionspreis der amerikanischen Steinkohle, auf SKE umgerechnet, geringer ist als der der europäischen Steinkohle. Außerdem kommt die Hauptmasse der amerikanischen Steinkohle in geologisch besonders günstiger Form und nahe der Erdoberfläche vor, deren Produktion daher eine geringere Kapitalmasse erfordert, als vergleichsweise die éuropäische Steinkohle, die in geologisch weniger günstiger Form und vergleichsweise tiefer unter der Erdoberfläche vorkommt als die amerikanische. Hinzu kommt noch die höhere Produktivkraft der Arbeit im US-amerikanischen Bergbau gegenüber jener der europäischen Bergarbeiter, die aus dem Kapital selbst entspringt. Das technologische Niveau der Produktion und damit die organische Zusammensetzung des amerikani-

11) Die Förderleistung des Rohöls pro Sonde und Tag beträgt z. B. in den USA durchschnittlich nur zwei Tonnen, im Nahen Osten beträgt sie aber je nach Ölquelle zwischen 500-1900 Tonnen. 
schen Steinkohlenbergbaus ist höher als das europäische Niveau der Technik in derselben Produktionssphäre.

Der durchschnittliche individuelle Produktionspreis der europäischen Steinkohle ist so hoch, daß sie durch die Konkurrenz der amerikanischen Steinkohle und in stärkerem Maße noch durch die des Rohöls schon längst vom Markt verdrängt worden wäre, wäre ihre Produktion nicht über die unmittelbare ,S Sozialisierung der Verluste" durch die Verstaatlichung der Sphäre wie in Frankreich und in Großbritannien bzw. über die mitteibare ,Sozialisierung der Verluste" durch die Subventionierung der Produktion in der Sphäre nicht aufrechterhalten worden. Die Stillegung zahlreicher Kohlenbergwerke und die Vernichtung von ungeheuren Kapitalmassen, also der gesellschaf tlichen Arbeit, mußte vorgenommen werden, weil sich das Kapital dort nicht durchschnittlich verwerten konnte, weil also der individuelle Produktionspreis der Einzelkapitale über dem allgemeinen, den Markt regulierenden Produktionspreis lag. Nur durch den politischen Eingriff des Staates und aus politischen Überlegungen wurde bisher in den wichtigsten europäischen steinkohleproduzierenden Staaten dafür gesorgt, daß nicht noch größere Teile der Produktionsanlagen stillgelegt wurden. Dagegen verwerten sich Kapitale, die in Amerika Steinkohle produzieren, durchschnittlich, realisieren also den marktmäßigen Durchschnittspronit.

Insofern reguliert der individuelle Produktionspreis der amerikanischen Steinkohle den Marktwert und den Marktpreis aller Energieträger, da ihr individueller Produktionspreis den Durchschnittsprofit gerade noch abwirft. Obwohl also andere Energieträger, wie die europäische Steinkohle, produziert und dem Energiemarkt zugeführt werden, deren individueller Produktionspreis noch höher ist als der individuelle Produktionspreis der amerikanischen Steinkohle, wird der Marktpreis für Energieträger nicht durch den individuellen Produktionspreis der europäischen Steinkohle bestimmt, da vom kapitalistischen Standpunkt die in dieser Sphäre angewandte Arbeit vergeudete Arbeit ist und daher von der kapitalistischen Gesellschaft nicht honoriert werden kann. Unter der Bedingung, daß die europäische Steinkohle durch staatliche Subventionen überhaupt produziert werden kann, wird der Marktpreis für Energieträger in der Sphäre so reguliert, als wäre die europäische Steinkohle auf dem Energiemarkt nicht existent.

Wollten wir den Tauschwertzusammenhang der Hauptenergieträger auf dem Weltmarkt, das Rohöl am Persischen Golf, das Rohöl in Amerika, die Steinkohle in Amerika und die Steinkohle in Europa veranschaulichen und die Relation des Marktpreises für Energie zum individuellen Produktionspreis der jeweiligen Energieträger, alle auf SKE umgerechnet, graphisch darstellen, so würde sich aufgrund der oben angeführten Analyse folgendes Bild ergeben (12):

12) Diese graphische Darstellung soll weniger die Verteilungsstruktur der Hauptenergieträger nach Art und Region, bzw. die Relation von deren individuellen Produktionspreisen zum regulierenden Marktpreis nach absoluten Größen genau wiedergeben, als vielmehr ihren tauschwertmäß̊gen Zusammenhang. Uns liegt hier sehr daran, zunächst die Zusammenhänge des Energiemarktes auf der Ebene der Abstraktion anschaulich zu machen. Dieses Bild dann aufgrund von genauen Angaben neu zu rekonstruieren, wäre ein empirisches Froblem. 


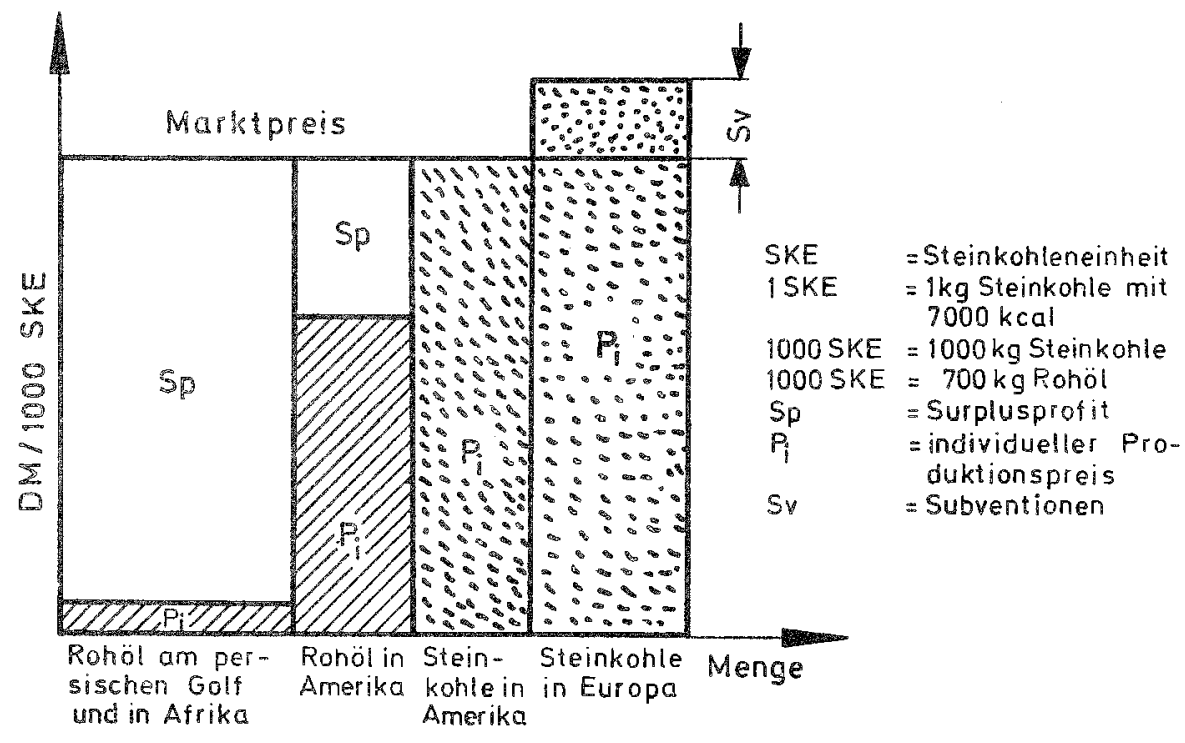

Wie aus der Abbildung 3 hervorgeht, ist die Produktivkraft der Arbeit auf dem Energiesektor am Persischen Golf am höchsten, die im europäischen Steinkohlenbergbau am niedrigsten, da die stoffliche Naturbasis der Energieträger am Persischen Golf am günstigsten, jene in Europa am ungünstigsten ist. Die Produktivkraft der Arbeit in der Sphäre ist an eine Naturbasis gebunden und, wie wir unter 2.1 ausführten, kann daher in der Sphäre nicht verallgemeinert werden. Kapitale, die die höhere Produktivkraft der Arbeit am Persischen Golf anwenden, erzielen ungeheure Massen von Surplusprofit (13). Wir haben unter 2.1 festgestellt, daß der Konkurrenz in der extralstiven Sphäre dadurch, daß die höhere, auf der günstigeren Naturbasis beruhende Produktivkraft der Arbeit nicht verallgemeinert werden kann, Schranken gesetzt sind, daß also die in der Sphäre angewandte gesellschaftlich notwendige Arbeit, damit also der Marktwert und der Marktpreis, nur in dem Maße sinkt, wie die durchschnittliche, aus dem Kapital selbst entspringende Produktivkraft der Arbeit in der Sphäre gesteigert werden kann. Das Wertgesetz erfährt also insofern die erste Modifikation als die Produktivkraft der Arbeit in der Sphäre auf einer Naturbasis beruht.

Auf den ersten Blick scheint aber nun die wirkliche Entwicklung in der Energiesphäre diesem allgemeinen ökonomischen Gesetz, der ersten Modifikation des Wertgesetzes in der extraktiven Sphäre also, zu widersprechen. Denn der Marktpreis für die wichtigsten Energieträger, auf SKE umgerechnet, ist über lange Zeiträume,

13) Auf die Frage der Aneignung dieses Surplusprofits kommen wir noch später zurück. 
und zwar seit den 50er Jahren bis vor kurzem, konstant geblieben (14), real aber im Hinblick auf die allgerneine Geldentwertung sogar gesunken, obwohl, wie aus der graphischen Darstellung der Abbildung 1 und der folgenden Tabelle 1 hervorgeht, das gesellschaftliche Bedürfnis nach Energie gewaltig gestiegen ist.

Vom marxistischen Standpunkt bedeutet diese Entwicklung eine gewaltige Steigerung der Produktivkraft der Arbeit, eine solche, die, wie wir noch sehen werden, nicht aus dem Kapital selbst entspringen kann. Sie deutet aber auch auf eine Verschärfung der Konkurrenz in der Sphäre hin. Wie kommt es also zu dieser Entwicklung; ist die erste Modifikation des Wertgesetzes in der Energiesphäre aufgehoben? Und welches sind die Bedingungen dieser Aufhebung? Die folgende Tabelle (14a) beinhaltet selbst eine Teilantwort auf diese Frage.

Der Weltenergicverbraucla, ${ }^{a}$, 1958 und 1967

\begin{tabular}{|c|c|c|c|c|c|}
\hline \multirow{2}{*}{ Winergieträger } & \multicolumn{2}{|c|}{ r958 } & \multicolumn{2}{|c|}{${ }_{0}^{1967}$} & \multirow{2}{*}{$\begin{array}{c}\text { Jahresdurch- } \\
\text { schnituliche } \\
\text { Veränderung } \\
\text { ro58/67 } \\
(v \mathrm{H})\end{array}$} \\
\hline & Mill $\mathfrak{t}$ & vII & Mill. $\ell$ & VHI & \\
\hline $\begin{array}{l}\text { Weltencrgicsesbranch. . . . . } \\
\text { davon: }\end{array}$ & 3713 & 100 & 5614 & 100 & +4.7 \\
\hline Ieste Brennstofic. . . . . . & 1991 & 53,6 & 2173 & 38,8 & $+1,0$ \\
\hline Flüssige Drennstofic. . . . . & I 139 & $3^{0,7}$ & 2223 & 39,6 & +7.7 \\
\hline Naturgas ......... & 508 & 13.7 & I 088 & 19.4 & $+8,9$ \\
\hline Elektrizität . . . . . . . & 76 & 2,0 & I 30 & 2,3 & $+6,2$ \\
\hline \multicolumn{6}{|c|}{$\begin{array}{l}\text { a Welt ohe sozialistische Lander (Osteuropa, UdSSK whd VR China). - b Gemessen in Mill. t Kohle- } \\
\text { aquivalmten. }\end{array}$} \\
\hline
\end{tabular}

Wie hieraus und aus der Abbildung $1 \mathrm{zu}$ sehen ist, hat sich mit dem ständigen Ansteigen des Weltenergiebedarfs auch die Verteilungsstruktur unter den Hauptenergieträgern ständig gewandelt. Der Anteil_der festen Brennstoffe, also der Steinkohle, an der Wéltenergieproduktion ist ständig gesunken, ihre absolute Produktion fast konstant geblieben und der Anteil der flüssigen Brennstoffe, also des Rohöls, ständig gestiegen. Unter den die verschiedenen Energieträger produzierenden Kapitalen hat also eine verschärfte Konkurrenz stattgefunden, als deren Folge die rohölproduzierenden Kapitale ihren Marktanteil an der Gesamtenergieproduktion, in SKE umgerechnet, zu Lasten der steinkohleproduzierenden Kapitale erheblich vergrößern konnten.

Die erste Modifikation des Wertgesetzes in der Energiesphäre ist also in der Tat dadurch aufgehoben, daß jene Kapitale, die die höhere Produktivkraft der Arbeit, weil die gïnstigere Naturbasis der Produktivkraft, anwenden, ihren Marktanteil erheblich vergrößerten. Vom Standpunkt des Wertgesetzes ist die Wirkung der Vergrößerung des Marktanteils der die höhere Produktivkraft anwendenden Kapitale in der Sphäre dieselbe, als wäre die durchschnittliche Produktivkraft der Arbeit selbst in der Sphäre gesteigert worden. Die Folge davon ist, wie wir in der Energie14) Die Weltwirtschaft. Tübingen 1970 , Heft 1 , S. 81

14a) Quelle: UN, Statistical Yearbook, New York 1967, S. 63; 1968, S. 63, übernommen aus: Die Weltwirtschaft, Tübingen 1970, Heft 1, S. 81 
sphäre jahrzehntelang beobachten konnten, die tendenzielle Senkung des Marktpreises, weil infolge der ständigen Senkung der durchschnittlichen gesellschaftlich notwendigen Arbeit in der Sphäre die tendenzielle Senkung des Marktwertes.

Während auf der einen Seite also der allgemeine, den Markt regulierende Produktionspreis in der Energiesphäre gesunken ist, ist der individuelle Produktionspreis jener die Steinkohle produzierenden Kapitale konstant geblieben bzw. zumindest in Europa nicht so weit gesunken, daß sie sich auch ohne die unmittelbare bzw. mittelbare Subventionierung durch den Staat (wie in England, Frankreich und in der BRD) hätten durchschnittlich verwerten können (vgl. Abb. 3).

Damit ist aber die oben gestellte Frage nur zum Teil beantwortet worden. Denn die natürlichen Quellen des Rohöls als die z. Z. günstigste Naturbasis der Produktivkraft der Arbeit in der Sphäre sind einerseits beschränkt, eine Beschränktheit, die heute so konkret ist wie die Beschränktheit keines anderen natürlichen Rohstoffes. Andererseits sind die Rohstoffquellen der Welt einschließlich der Rohölquellen, in welcher Form und wie auch immer, unter das Grundeigentum subsumiert. Es ist klar, daß die besondere Form des Grundeigentums an Rohstoffquellen nicht das Privateigentum, sondern in der Regel das Gemeineigentum ist. Die herrschende Klasse insgesamt ist also jeweils die mittelbare Grundeigentümerin der Rohstoffquellen, deren Verhältnis zum Grundeigentum vermittels des diese Klasse repräsentierenden Staates ge regelt wird (15).

Was aber das Verhältnis des Grundeigentums zum Kapital anbetrifft, ist es lediglich die Form, worin sich das private Eigentum an Rohstoffquellen vom Gemeineigentum an denselben unterscheidet, genau so wie es lediglich eine Formveränderung ist, wenn das Privateigentum an agrikulturellem Grund und Boden in das Staatseigentum übergeht.

„Das Grundeigentum wird so von zwei Seiten her negiert; die Negation von seiten des Kapitals ist nur Formveränderung, zu seiner Alleinherrschaft. (Grundrente als die allgemeine Staatsrente (-steuer), so daß die bürgerliche Gesellschaft in anderer Weise das mittelaltrige System, aber als die vollständige Negation đesselben, reproduziert.) ${ }^{6 /}$ (16)

Die Formveränderung des Grundeigentums und die Verwandlung des Privateigentums in das Eigentum des das Kapital repräsentierenden Staates bedeutet also keineswegs die Aufhebung des besonderen Verhältnisses des Grundeigentums zum Kapital. Die Grundrente verwandelt sich unter dieser Bedingung in die ,allgemeine Staatssteuer", sie wird jedoch nicht aufgehoben. Und wir haben an einer anderen Stelle gesehen, daß dieses besondere Verhältnis, in dem das Grundeigentum unmittelbar als Schranke des Kapitals fungiert, nur dann vollständig aufgehoben werden kann, wenn die Beschränktheit des Grund und Bodens und der Rohstoffquellen

15) Diese Aussage bezieht sich nicht auf die europäischen Gesellschaften, wo das Privateigentum am Grund und Boden (einschließlich der Rohstoffquellen) das herrschende vorkapitalistische Eigentumsverhältnis war, sondern sie bezieht sich insbesondere auf die orientalisch-asiatischen Gesellschaften, wo das Gemeineigentum am Grund und Boden das herrschende vorkapitalistische Eigentumsverhältnis war und wo der Übergang zur kapitalistischen Produktionsweise auch ohne die Entfaltung des Privateigentums zum herrschenden Grundeigentumsverhältnis erfolgte.

16) Marx, Karl: Grundrisse der Kritik der politischen Ökonomie. Berlin 1953, S. 190 
vollständig aufgehoben ist (17).

Bei Beschränktheit der unmittelbar von der Natur vorgegebenen Produktionsbedingungen und unter der Voraussetzung, daß diese Produktionsbedingungen unter das Gemeineigentum subsumiert sind, verhält sich der Eigentümerstaat einer Gesellschaft dem Kapital gegenüber wie der wirkliche Eigentümer, obgleich dieser seinem Wesen nach ais der ideelle Gesamtkapitalist derselben Gesellschaft fungiert.

Es ergibt sich demnach von selbst, daß auf der Weltmarktebene das Grundeigentum, wenn auch als Staatseigentum, dem auf ihm produzierenden Kapital erst recht Schranken setzen muß, wenn der als der ideelle Gesamteigentümer auftreten. de Staat weit davon entfernt ist, zugleich auch als der unmittelbare Repräsentant jenes Kapitals zu erscheinen.

Wie kommt es aber, daß in der Praxis auf dem Energiesektor das Grundeigentum an Rohölquellen nicht als Schranke des Kapitals fungiert, also dem Konkurrenzkampf der Einzelkapitale in der Sphäre, die Produktion von Rohöl zu Lasten der Steinkohle verstänt zu steigern, keine Schranken setzt? Ferner ist zu fragen, unter welchen Bedingungen sich das Grundeigentum durch die Modifikation des Wertgesetzes, wie oben unter 2.2 abgeleitet wurde, als solches nicht verwirklichen kann.

Dieser Fragenkomplex kann allerdings nur im Zusammenhang mit dem besonderen Verhältnis des Kapitals zum Grundeigentum einerseits und dem besonderen historischen Charakter des Grundeigentums selbst andererseits beantwortet werden.

\section{Das historische Verhältnis des Kapitals zum Grundeigentum}

Es sind dem Wesen nach zwei Formen des Grundeigentums zu unterscheiden: Die alte Form des Grundeigentums in der vorkapitalistischen und die moderne Form desselben in der bügerlichen Gesellschaft:

„Sowohl seiner Natur nach, wie historisch ist das Kapital der Schöpfer des modernen Grundeigentums, der Grundrente; wie seine Aktion daher auch als Auflösung der alten Form des Grundeigentums erscheint. Die neue entsteht durch die Aktion des Kapitals auf die alte" (18).

Mit dem Grundeigentum als Schranke des Kapitals in unseren obigen Ausfürungen war selbstverständlich immer dessen moderne Form gemeint. Denn erst durch die Entwicklung der kapitalistischen Produktionsweise in den unter das Grundeigentum subsumierten Produktionssphären ist das Grundeigentum uberhaupt in der Lage, einen Teil des produzierten Mehrwerts als Grundrente abzuzweigen:

"In demselben Maßs, wie sich mit der kapitalistischen Produktion die Warenproduktion entwickelt, and daher die Produktion von Wert, entwickelt sich die Produktion von Mehrwert und Mehrprodukt. Aber in demselben Maß, wie letztre sich entwickelt, entwickelt sich die Fähigkeit des Grundeigentums, einen wachsenden Teil dieses Mehrwerts, vermittelst seines Monopols an der Erde abzufangen, daher den Wert seiner Rente zu steigern und den Preis den Bodens selbst's (19).

17) Massarrat, M.: Zur Problematik der gleichen Mehrwertrate ..., a. a. O., (vgl. Fußnote 2)

18) Marx, Karl: Grundrisse ..., a. a. O., S. 187

19) Marx, Karl: Das Kapital ..., a. 2. O., Bd. III, S. 651 
Das Kapital und das moderne Grundeigentum stehen in einem dialektischen Verhältnis zueinander. Das Kapital, selbst als Produkt der Zersetzung der alten Grundeigentumsverhältnisse, schafft das moderne Grundeigentum und verwandelt es in seinen Gegensatz:

"Das Kapital geht aus der Zirkulation hervor und setzt die Arbeit als Lohnarbeit; bildet sich so aus, und als Ganzes entwickelt, setzt (es) das Grundeigentum, sowohl als seine Bedingung wie als seinen Gegensatz ${ }^{66}(20)$.

Das moderne Grundeigentum mit der kapitalistischen Gesellschaft insgesamt einmal national voll entwickelt, muB als Schranke des Kapitals aufgehoben werden, kann sich das $\mathbb{K}$ apital selbst historisch als ein noch entwicklungsfähiges gesellschaftliches Verhältnis durchsetzen.

In der Tat hebt auch das Kapital das Grundeigentum als seine Schranke zunächst auf nationaler Ebene auf. Dieser Prozeß erfolgt dadurch, daß das Kapital die Reschränkthei der unmittelbar von der Natur vorgegebenen Produktionsbedingungen historisch aufhebt, indem es die nationalen Schranken überwindet, erstmalig also den kapitalistischen Weltmarkt - zu unterscheiden vom bereits herausgebildeten Handelsweltmarkt - schafft, sich also dorthin bewegt, wo Grund und Boden und Rohstoffquellen in relativ unbeschränktem Umfang existieren. Sind Grund und Boden sowie Rohstoffquellen auf intemationaler Ebene in relativ unbeschränktem Umfang vorhanden, so hört das nationale Grundeigentum auf, wie unter 2.3 angeführt, als Schranke des Kapitals zu fungieren.

Indes stößt das Kapital, das nationale Grundeigentum als seine Schranke aufhebend, als historisch fertiges Produkt international auf die alte vorkapitalistische Form des Grundeigentums, das es sich zunächst unterwerfen muß, soll das Kapital verhindern, daß anstelle des nationalen Grundeigentums nun das fremde Grundeigentum als seine Schranke auftritt. Insofern ist die Subsumtion des internationalen Grundeigentums unter das Kapital als historische Bedingung seiner Entfaltung die Kehrseite der Negation des nationalen Grundeigentums als sein Gegensatz.

Der historische Zeitraum dieses Verwandlungsprozesses des Kapitalverhältnisses zum Grundeigentum ist $u$. $\mathbb{E}$. die zweite Hälfte des 19. Jahrhunderts, in dem das vorkapitalistische Grundeigentum im internationalen Umfang unmittelbar durch Annexion bzw. Kolonisation oder aber mittelbar durch die Eingliederung der auf dem Grundeigentum beruhenden ökonomie in den Weltmarkt unter das Kapita! subsumiert wird (21).

Das Kapital ist unter der Bedingung des besonderen Verhälînisses zum Grundeigentum in der Lage, die von der Natur vorgegebenen relativ unbeschränkten Produktionsbedingungen, also den agrikulturellen Grund und Boden sowie die mineralischen Rohstoffquellen, uneingeschränkt anzuwenden, und zwar jene, die dessen höchste Verwertung erwarten lassen, ohne den gesamten erzielten Surplusprofit an das Grundeigentum abgeben zu müssen.

20) Marx, Karl: Grundrisse ..., a. a. O., S. 189

21) Dieser historische Verwandlungsprozeß wird in einer noch nicht abgeschlossenen Untersuchung über „Die politische Ökonomie der Entwicklung und Unterentwicklung im internationalen System " vom Verfasser näher ausgeführt und begründet. 
Dex Profit des Kapitals wird insofern beschnitten, als es einen Teil des erzielten bzw. des zu erwartenden Mehrwerts zur Herstellung der allgemeinen Produktionsbedingungen anwenden muß. Hierzu gehören nicht nur eine für die kapitalistische Produktion unumgängliche Infrastruktur und Verwaitung, sondern die Kosten für die Reproduktion der auf den vorkapitalistischen Grundeigentumsverhältnissen beruhenden Herrschaftsformen. Denn das besondere Verhältnis des Kapitals zum Grundeigentum, in dem das letztere nicht als Schranke des ersteren fungiert, kann nur solange bestehen, wie die vorkapitalistischen Grundeigentums- und Klassenverhältnisse aufrechterhalten bleiben können.

Die herrschenden Klassen der vorkapitalistischen Gesellschaften ihrerseits als die mittelbaren Gesamteigentümer des nationalen Grund und Bodens und der nationalen Rohstoffquellen dieser Gesellschaften geben sich damit, daß das Kapital ihr Grundeigentum als die Naturbasis seiner Produktion anwendet, mit einem Tribut gänzlich zufrieden, mil einer Art vorkapitalistischer Grundrente also, auf der ihre Macht und Herrschaft seit eh und je beruhte.

Es ist klar, daß dieser Tribut, diese Grundrente, mit der vom Kapital selbst geschaffenen kapitalistischen Grundrente nicht das geringste zu tun hat und daß dieser Tribut des Kapitals an das Grundeigentum nur einen kleinen Teil der eigentlichen kapitalistischen Grundrente, des vom Kapital insgesamt erzielten Surplusprofits also, ausmacht. Insofern fungiert das vorkapitalistische Grundeigentum nicht als Schranke des Kapitals, schließt also keineswegs Grund und Boden bzw. die Rohstoffquellen von der Produktion aus, um die Grundrente hochzuschrauben. Daher können Kapitale in der Sphäre der Rohstoffproduktion einschließlich der Produktion von Energieträgern miteinander konkurrieren, indem sie durch die Anwendung von immer günstigerer Naturbasis ihrer Produktion in den vorkapitalistischen Gesellschafter - vorausgesetzt, daß die günstigere Naturbasis der Rohstoffproduktion auch in der Tat in den vorkapitalistischen Gesellschaften in ausreichenderm Umfang vorhanden ist - die von ihnen angewandte Produktivkraft der Arbeit zu steigern und ihren individuellen Produktionspreis herabzudrücken suchen.

Damit ist also klar, weshalb in der Sphäre der Energieproduktion, obgleich die Naturbasis der Produktion beschränkt und unter das Grundeigentum subsumiert, diese dem Kapital als unbeschränkt erscheint und weshalb also in der Sphäre eine Konkurrenz staitfinden und das Wertgesetz wirken kann.

Es ist ebenso klar, weshalb einige Jahrzehnte lang nicht das Grundeigentum, und im Fall der Rohölproduktion also die Rohölförderländer, sondern das Kapital, also die multinationalen ölkonzerne, über die zu produzierende Menge, über den Marktpreis und selbst über die Höhe der dem Grundeigentum zufallenden Grundrente bestimmen konnte. Die Frage ist nun, wie und unter welchen Bedingungen sich seit Beginn der 70er Jahre eine deutliche Tendenzwende im Verhältnis des Kapitals zum Grundeigentum im Falle der Rohölproduktion abzuzeichnen begann.

Wis haben oben ausgeführt, daß das Grundeigentum dem Kapital nur als dessen vorkapitalistische Form unterworfen bleibt, also nicht als Schranke des letzteren fungiert. Von diesem Standpunkt kann das unmittelbare Interesse des Kapitals, die auf dem Grundeigentum beruhenden vorkapitalistischen Herrschaftsformen aufrechtzuerhalten, abgeleitet werden. Dieses unmittelbare Interesse des Kapitals 
reflektiert sich auch in der Tat in der jahrzehntelangen bedingungslosen Unterstützung despotischer Herrschaftsformen der rohstoffeichen vorkapialistischen Gesellschaften durch den Imperialismus.

Dem Kapital war zwar eine Zeitlang möglich, die auf vorkapitalistischen Grundeigentumsverhältnissen beruhenden Herrschaftsformen mit politischen Eingriffen und militärischer Gewalt zu erhalten, wenn diesen die Gefahr drohte, durch den beginnenden Auflösungsprozeß alter Verhältnisse beseitigt zu werden. Was aber das Kapital nicht schafft, ja auch nicht schaffen kann, ist, die Auflosung der vorkapitalistischen Produktionsverhältnisse und die Entwicklung der kapitalistischen Mehrwertproduktion zu verhindern, weil das Kapital selbst die eigentliche Ursache der Zersetzung des vorkapitalistischen Grundeigentums und der unmittelbare Träger der kapitalistischen Produktionsweise in den vorkapitalistischen Gesellschaften ist.

Wie das Kapital um die Jahrhundertwende des 18. zum 19. Jahrhunderts in die Agrikultur Englands, später in die der Kolonien eindrang, das vorkapitalistische Grundeigentum zunächst als seine Bedingung zersetzte und dieses in seine Gegensatz verwandelte - um die historische Parallele dazu aufzuzeigen - zersetzt das Kapital iber 150 Jahre später in internationalem Umfang das vorkapitalistische Grundeigentum rohstoffreicher Gesellschaften und verwandelt dieses zwangsläufig auch in seinen Gegensatz.

Hat sich einmal die kapitalistische Mehrwertproduktion als die bestimmende Form der Produktion in den ehemals vorkapitalistischen Gesellschaften durchgesetzt und haben sich dort die Klassenverhältnisse grundlegend verwandelt, dann hört die herrschende Klasse auf, sich dafür, daß sie dem Kapital die Naturbasis der Produktion von Rohstoffen, als deren Eigentümerin sie auftritt, zur Verfügung stellt, mit dem bloßen Tribut des Kapitals zufriedenzugeben, wo nun ihre eigene Macht auf der Produktion von Mehrwert beruht. Sie beginnt, der wahllosen Ausbeutung nationaler Rohstoffe durch das Kapital, zumal fremdes Kapital, dadurch also dem Konkurrenzkampf der Kapitale in einer Sphäre, die Produktivkraft der Arbeit durch die beliebige Anwendung günstigerer Naturbasis zu steigern, ein Ende zu setzen und auf diese Weise auch auf die Gestaltung des Marktpreises in der Sphäre Einfluß zu nehmen.

Die herrschenden Klassen dieser Gesellschaften beginnen, als faktische Eigentïmer der Rohstoffquellen, die Höhe des ihnen gezahlten Tributs selbst zu bestimmen und den Tribut so weit hochzuschrauben, wie es ihnen auch als Grundeigentümer im kapitalistischen Weltsystem in Wirklichkeit zusteht. Es handelt sich um den gesamten vom Kapital aufgrund der Anwendung von günstigerer Naturbasis der Produktion erzielten Surplusprofit. Die Konkurrenz der Kapitale um die Erwerbung yon besonders günstiger Naturbasis der Rohstoffproduktion, falls sie unter ganz bestimmien politischen Bedingungen und mit Hilfe von politischen Eingriffen nicht ausgeschaltet wirde, wird dafür sorgen, daß der gesamte, durch die Anwendung dieser Naturbasis erzielte Surplusprofit sich in die kapitalistische Grundrente verwandelt und vom Grundeigentümerstaat angeeignet wird.

Was das Verhältnis des Kapitals zum Grundeigentum auf dern Energiesektor betrifft, so hat der oben dargestellt Wandlungsprozeß vom alten Verhältnis des Kapitals zum vorkapitalistischen Grundeigentum zu einem neuen Verhältnis des 
Kapitals zum von ihm selbst geschaffenen modernen Grundeigentum schon längst begonnen, dessen Folgen wir genau jetzt erieben.

Die Tendenzwende in dern Verhallnis der multinationalen Ölkonzerne zu den rohölreichen Gesellschaften ist also weder das Resultat der besonders klugen Politik einiger Harvard-Absolventen in den ölforderländern noch die Folge der schlechten Außenpolitik der USA, noch ist der letzte Nahostkrieg selbst die eigentliche Ursache dieser Iendenzwende, wie seitens der bürgeriichen Presse und der Politiker vereinfacht dargestellt wird, sondern sie ist das Resultat der kapitalistischen Entwicklung in den rohölreichen Gesellschaften selbst. Der Nahostkrieg hat die Tendenzwende allenfalls beschleunigt.

Hieraus kann allerdings nicht gefolgert werden, daß die kapitalistische Proăuktionsweise sich in allen diesen Gesellschaften schon durchgesetzt haben muB, wie auch daraus nicht gefolgert werden kann, daß diese Gesellschaften mit der Entwicklung des nationalen Kapitalismus bereits ökonomisch und politisch völlig selbständig geworden sind. Ferner kann daraus auch nicht geschlossen werden, daß die kapitalistisch am weitesten entwickelte Gesellschaft in dem Umbruchsprozeß der Beziehungen zwischen den multinationalen ölkonzernen und den Rohölförder* ländern automatisch die führende politische Rolle haben muß. Es spielen zu viele Einflußfaktoren eine Rolle, als daß das Verhältnis der Ökonomie zur Politik auf dieses einfache Verhältnis reduziert werden könnte.

Zu diesen Einflußfaktoren gehören z. B. die besondere innere politische Struktur, der politische und militärische Abhängigkeitsgrad vom Imperialismus, die Abhängigkeit der übrigen Sektoren der nationalen Wirtschaft vom Weltmarkt etc. Ferner darf das besondere Kräfteverhältnis der sozialistischen Staaten einerseits und der kapitalistischen Staaten andererseits als reales Machtverhältnis nicht unberück. sichtigt bleiben, ein Faktor, der allerdings nicht allein auf die Funktion eines einzigen Staates in diesem Verwandlungsprozeß bestimmend wirkt, sondern auf die Tendenzwende im Verhältnis der ölförderländer zu den multinationalen Konzernen insgesamt. Dieses Kräfteverhältnis und die reale Macht der sozialistischen Staaten hat zwar diese Tendenzwende nicht verursacht und auch nicht ausgelöst, sondern dahingehend begünstigt, als es die Verschiebung dieser Tendenzwende durch militärische Gewalt seitens des Imperialismus von vornherein ausschloß.

Libyen, als ein unter den rohölfördernden Ländern kapitalistisch am wenig. sten entwickeltes Land, hat neben Algerien bei der Herbeiführung der Tendenzwende im Verhältnis der multinationalen Konzerne zu den rohölfördernden Ländem zweifellos nicht nur in der Preispolitik die führende Rolle gespielt, während dem Iran, als dem unter diesen Ländern kapitalistisch am weitesten entwickelten Land, neben Saudi-Arabien bisher in diesem Prozeß die Bremserrolle zukommt (22).

22) Charakteristisch für die Rolle Libyens und des Iran in diesem Zusammenhang ist die Freisentwicklung für Rohöl seit 1971. ,Bis zum Teheraner Beschlub, den Listenpreis pro Barrel (159 Liter) Rohöl auf 11,65 Dollar reichlich zu verdoppein, hatten die libyschen Steuerreferenzpreise am stärksten angezogen: dort erhöhten sie sich je Barrel von 2,55 Dollar in 1971 auf 8,925 Dollar am 1.11.1973. In der gleichen Zeit stieg der Barrel-Preis fur venezolanisches 01 von 2,09 Dollar auf 7,261 Dollar, für nigerisches ôl von 2,42 Dollar auf 8,31 Dollar, für arabisches 01 von 2,55 Dollar auf 5,17 Dollar und für persisches of won 1,72 Dollar auf 5,046 Dollar." Frankfurter Rundschau vom 29.12.1973. 
Es ist klar, daß Libyen nur deshalb die führende Rolle in den Auseinanderseto zungen der OPEC-Staaten mit den Ökonzemen spielen konnte, weil die objektiven Bedingungen für eine Tendenzwende in den wichtigsten OPEC-Staaten schon gegeben waren, diese Staaten also über kurz oder lang selbst ähnliche Schritte hätten un ternehmen müssen, was schon in der Gründung der OPEC selbst, als die institutionelle Bedingung einer notwendigen Veränderung des alten Verhältnisses der multinationalen Konzerne zu den Ölförderländern, zum Ausdruck kommt. Hätte nämlich diese libysche Regierung schon vor der Gründung der OPEC dieselben Maßnahmen getroffen, wie seit 1971, so wäre sie diesen aller Wahrscheinlichkeit nach selbst zum Opfer gefallen.

Das historizche Beispiel dafür liefert uns der erste, offensichthich bereits vergessene, Versuch des nationalen Bürgertums in Iran, schon 1951 die erst in der Gegenwart erfolgte Tendenzwende im Verhältnis des Weitkapitals zum Grundeigen. tum herbeizufuhren. Als die Auflösung der vorkapitalistischen Eigentums- und Froduktionsverhältnisse im Iran sehr weit vorangeschritten war und das nationale Bürgertum unter der Führung von Dr. Mossadegh mit der Verstaatlichung der gesamten Ölproduktionsanlagen der BP im Iran den Imperialismus und seinen damaligen einheimischen Verbündeten, die iranische Großgrundbesitzerklasse, empfindlich zu treffen drohte, war der Imperialismus deshalb in der Lage, durch den organisierten Wirtschaftsboykott von außen und die Verschwörung von innen -- ähnlich wie im Fall Chile, allerdings auf einer anderen qualitativen Ebene - die Machtibernahme durch das nationale Bürgertum zu verhindern und damit diesen ersten Versuch $z u$ vereiteln, weil die objektiven Bedingungen in den übrigen ölproduzierenden Staaten für die Veränderung des Verhältnisses. des Weltkapitals zum Grundeigentum zu diesem Zeitpunkt noch weitgehend fehlten.

Bevor nun dieser Abschnitt abgeschlossen wird, soll noch auf die logische Konsequenz der historischen Entwicklung des Verhältnisses des Kapitals zum Grundeigenturn eingegangen werden. Hierzu ist das in diesem Zusammenhang bereits gesagte zunächst zusammenzufassen: Das Kapital selbst, als Produkt der Zersetzung der vorkapitalistischen Grundeigentumsverhältnisse, erst national herausge. bildet, schafft das moderne Grundeigentum auf nationaler Ebene und verwandelt dieses, obgleich ursprünglich seine Bedingung, in seinen Gegensatz. Diesen Gegensatz hebt das nationale Kapital auf, indem es sich zum Weltkapital machtund als Kehrseite dieser Aufhebung sich das vorkapitalistische Grundeigentum im internationalen Umfang unterwirft. Somit reproduziert sich historisch das ursprüngliche Verhältnis des Kapitals zum Grundeigentum, aber diesmal im internationalen Umfang und mit dem Unterschied, dab hier, weil das Kapital bereits als fertiges Produkt auftritt, das vorkapitalistische Grundeigentum nicht die Bedingung seiner Entstehung, sondern die Bedingung seiner Weiterentwicklung darstellt. Die Weiterent-

Doch auch dem Teheraner Reschluß, den Ölpreis auf 11,65 Dollar pro Barrel zu erhöhen, war kurz zuvor eine Rohölpreiserhöhung durch Libyen auf über 15 Dollar voraugegangen. Während Libyen unter den OPEC-Staaten den Ölkonzernen immer als erstes Forderungen stellte und den Rohölpreis heraufsetzte, die anderen OPECStaaten also immer nachziehen mußten, befleißigte sich der Schah ständig mit Maßhalteappellen und ging selbst, wie die Frèisentwicklung für das persische Öl eindeutig zeigt, mit, gatem Beispiel" voran. 
wicklung des Kapitals im internationalen Umfang setzt aber die Zersetzung der vorkapitalistischen Grundeigentumsverhältnisse im internationalen Umfang voraus, ein Prozeß, der sich in unserer Gegenwart beschleunigt vollzieht.

Setzt sich die kapitalistische Produktionsweise in allen vorkapitalistischen Gesellschaften einmal durch, verwandelt sich die alte vorkapitalistische Form des internationalen Grundeigentums in die moderne Form desselben, so reproduziert sich historisch der alte Gegensatz des Grundeigentums zum Kapital, aber diesmal im internationalen Umfang. In der Konsequenz dieses historischen Verhältnisses des Kapitals zum Grundeigentum liegt es auch, daß dieser Gegensatz, weil er dann voll entwickelt ist, nur mit dem Kapitalverhältnis selbst aufgehoben werden kann.

\section{Die Aneignung des Surplusprofits auf dem Energiesektor}

Die allgemeine Verwirrung, die über die Mechanismen der Preisbestimmung auf dem Energiesektor herrscht, und die gezielte Propaganda von bestimmten bürgerlichen Massenmedien haben den Eindruck entstehen lassen, als würde der Rohölpreis erstens von den ölförderländern und zweitens willkürlich festgesetzt. Wir haben aber oben gesehen, daß der Preis für Rohöl in einem unmittelbaren Zusammenhang mit dem Preis anderer Energieträger steht und daß dieser Preis durch die Konkurrenz der Kapitale in der Sphäre, und zwar von den steinkohleproduzierenden Kapitalen, bestimmt wird. Es ist andererseits auch bekannt, daß es sich bei den in der Energiesphäre im Konkurrenzverhältnis stehenden Kapitalen nicht um die Konkurrenz der nationalen Kapitale der Ölförderländer im Nahen Osten mit den Kapitalen handelt, die in Europa oder Amerika andere Formen von Energieträgern produzieren, sondern, daß die gesamte Energiesphäre von einigen wenigen euro-amerikanischen Kapitalen, die in der Sphäre miteinander auch konkurrieren, beherrscht wird.

Die Oo̊lförderländer setzen, wie oben abgeleitet, unter bestimmten historischen Bedingungen der Konkurrenz der Kapitale in der Sphäre Schranken, also dadurch, daß sie im Verhältnis des Kapitals zum Grundeigentum als kapitalistisches Grundeigentum auftreten und der Konkurrenz der Kapitale in der Sphäre auch deshalb Schranken setzen können, weil die Rohölquellen nunmehr beschränkt werden. Wären nämlich die Rohölquellen unbeschränkt, so würde die Konkurrenz unter den Grundeigentümerstaaten, ihre Ölquellen zur Ausbeutung zu verpachten, dazu führen, daß das Grundeigentum als Schranke des Kapitals aufgehoben wird.

Wie dem auch sei, haben weder die Staaten der Ölförderländer irgendeinen Einfluß auf die Gestaltung des Marktpreises für Rohöl, solange sie als bloße Eigentijmer der Ölquellen auftreten, noch steht der Marktpreis des Rohöls als Energieträger in irgendeinem Zusammenhang mit dessen individuellem Produktionspreis, solange ungünstigere Arten von Energieträgern, wie die Steinkohle, in bedeutendem Umfang produziert werden. Die eigentliche Funktion der Ölförderstaaten, solange sie als Bloße Verpächter auftreten, besteht darin, einen Teil der von den kapitalistischen Pächtern, den multinationalen Konzernen also, erzielten ungeheuren Massen von Surplusprofit als Grundrente (genauer Differentialrente) abzuzweigen.

Man könnte aber zu Recht dagegen einwenden, daß die OPEC Staaten im 
Dezember 1973 in Teheran gerade eine Verdoppelung des ölpreises beschlossen haben und daß daher die Wirklichkeit der obigen These widerspricht. Denn auf der einen Seite haben wir begründet, weshalb der Marktpreis auch für Rohöl durch den individuellen Produktionspreis der Steinkohle bestimmt wird und auf der anderen Seite sehen wir aber hier, daß die Rohölförderstaaten den Rohölpreis erhöht haben. Wir werden im folgenden sehen, daß dies nur ein Scheinwiderspruch ist, daß also die Rohölförderstaaten in der Tat keinen unmittelbaren Einfluß auf die Gestaltung des wirklichen Marktpreises haben. Dennoch macht dieser berechtigte Einwand klar, daß die Problematik der Preisbildung auf dem Energiemarkt komplizierter ist, als wir im Abschnitt 3. darstellten.

Dort haben wir bewußt das Preisverhältnis der Steinkohle zum Rohöl auf das Verhältnis der in beiden Rohstoffen enthaltenen Energiemenge je Tonne reduziert, und zwar aus zwei Gründen: Erstens deshalb, weil für Rohöl zunächst und in der Hauptsache als Energieträger ein bestimmtes gesellschaftliches Bedürfnis existiert, Rohöl also einen Preis als Energieträger besitzt, der auch in der Tat von den multinationalen Konzernen für die Berechnung der Abgaben an die Ölförderstaaten zugrundegelegt wird. Zweitens deswegen, weil die Ableitung des Marktpreises auf dem Energiesektor, und vor allem die Darstellung des Tauschwertzusammenhangs in der Sphäre, dadurch sehr erleichtert wurde.

Der wirkliche Marktwert und Marktpreis für Rohöl wird aber nicht ausschließlich durch die Energiemenge, die, in SKE ausgedrückt, darin enthalten ist, bestimmt, sondern noch zusätzlich dadurch, um welchen Betrag die Kosten für die Gewinnung von modifizierten Energieträgern, wie Benzin, Heizöl etc., aus Rohöl geringer sind, als bei der Gewinnung derselben Energieträger aus der Steinkohle.

Nehmen wir an, der individuelle Produktionspreis von Steinkohle, in SKE umgerechnet, betrage Pi DM. Da Steinkohle und Rohöl zunächst lediglich Rohstoffe sind, erst also zur Verwandlung in Endprodukte verarbeitet werden müssen, bestimmen die Verarbeitungskosten der Steinkohle und des Rohöls zu Endprodukten die Konkurrenzfähigkeit der beiden Energieträger und die Substituierbarkeit des einen durch den anderen entscheidend. Es ist auch bekannt, daß die Verarbeitungskosten des Rohöls von durchschnittlicher Qualität zu Endprodukten um ein vielfaches geringer sind als jene der durchschnittlichen Steinkohle (23).

Nehmen wir also an, die Verarbeitungskosten für eine bestimmte Menge SKE, in Gebrauchswertform von Steinkohle umgerechnet, betrügen V DM. Der durchschnittliche Marktpreis für die aus Steinkohle gewonnenen Endprodukte, bezogen auf die Menge SKE, betrüge dann insgesamt $\mathrm{Pi}+\mathrm{V} \mathrm{DM}$.

Sind dagegen die Verarbeitungskosten derselben Menge SKE in Form von Rohöl zu Endprodukten x <V DM, dann ist der Marktpreis für Rohöl als Rohstoff für eine bestimmte Menge SKE um den Betrag von $V-x=Z$ DM höher als der Marktpreis bzw. der individuelle Produktionspreis Pi derselben Menge SKE in Form von Steinkohle als Rohstoff. Da also der Marktpreis für die Menge SKE durch den

23) Der endgültige Preis für das aus Rohöl gewonnene Benzin ohne die Steuern beträgt zwischen 10-15 Pfg. pro Liter. Dagegen beträgt It. einer Angabe des Spiegels (vom 7. 1. 1974, S. 79) der Preis für das aus Steinkohle gewonnene Benzin ebenfalls ohne Steuern zwi shen 35-45 Pfg. pro Liter. 
individuellen Produktionspreis von SKE in Form von Steinkohle (Pi) reguliert wird, würde der wirkliche Marktpreis von derselben Menge SKE in Form von Rohöl $\mathrm{Pi}+\mathrm{Z} \mathrm{DM}$ betragen, dies aber unabhängig davon, ob nun alle Endformen von Energieträgern, wie z.B. Benzin, Heizöl etc., auch in Wirklichkeit aus der Steinkohle gewonnen werden oder nicht. Denn der Marktpreis für Rohöl kann so weit nach oben steigen, wie er der Gesellschaft unter Berücksichtigung der geringeren Kosten seiner Verarbeitung gerade noch gegenüber der Steinkohle als eine Alternative erscheint. Die Gesellschaft ist also bereit, solange auf die Anwendung von Steinkohle als Energieträger zu verzichten, wie der Marktpreis für Rohöl als Energieträger unter $\mathrm{Pi}+\mathrm{Z}$ DM liegt, oder diesen Preis gerade noch erreicht (24).

$\mathrm{Zu}$ dem Marktpreis für Rohöl als bloßer Energieträger, der gegenüber der Steinkohle also erhebliche Vorteile auch bei der Verarbeitung aufweist, muß noch der Preis für die sog. Nebenprodukte hinzugezählt werden, die bei der Verarbeitung anfallen, für die aber in der chemischen und der pharmazeutischen Industrie eine große zahlungsfähige Nachfrage vorhanden ist. Wie hoch sich die Nebenprodukte, bezogen auf die Tonne Öl, verwerten, ist nicht bekannt, da die Mineralölkonzerne darüber wie über ihren wirklichen Gewinn und über ihre wirklichen Kosten nichts veröffentlichen. Tatsache ist aber, daß die Rohölnebenprodukte bei der Verarbeitung ein gesellschaftliches Bedürnnis befriedigen, daß sie also einen selbständigen Tauschwert besitzen, der im Marktwert und Marktpreis des Rohöls seinen Niederschlag finden muß. Wir nehmen an, daß der Wert und der Preis der Ölnebenprodukte, auf die SKE einer bestimmten Ölmenge umgerechnet, N DM beträgt. Demnach setzt sich der wirkliche, auf dem Energiemarkt auch in der Tat realisierbare Marktwert und Marktpreis einer Tonne des durchschnittlichen Rohöls (1 Tonne = 10000 SKE) aus folgenden Komponenten zusammen:

$$
\mathrm{Pi}+\mathrm{Z}+\mathrm{N}=\mathrm{M}_{\text {öl }} \mathrm{DM}
$$

$\mathrm{Pi}=$ Individueller Produktionspreis von 10000 SKE Steinkohle

$\mathrm{Z}=$ Der Differenzbetrag der Verarbeitungskosten von 10000 SKE Rohöl gegenüber den Verarbeitungskosten von 10000 SKE Kohle

$\mathrm{N}=$ Der Wert bzw. der Preis für die Ölnebenprodukte bezogen auf $10000 \mathrm{SKE}$ Rohöl

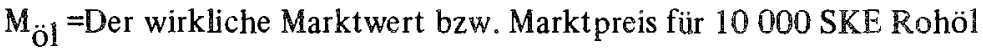

Wir werden sehen, daß auf dem Energiesektor in der Praxis zwei Preise für Rohöl existieren: zum einen der fingierte Rohölpreis (der sogenannte Listenpreis), der

24) Der individuelle Produktionspreis von SKE in Form von Steinkohle Pi muß nicht unbedingt mit dem wirklichen Produktionspreis von Steinkohle übereinstimmen. $P i$ ist in Europa z. B. der subventionierte Produktionspreis, der also geringer ist als der wirkliche Produktionspreis. Er erscheint aber auf dem Markt als der wirkliche Marktpreis der Steinkohle, weshalb auch der subventionierte Marktpreis (gleich dem individuellen Produktionspreis) der Steinkohle auf dem Markt als Vergleichsmaß mit dera Marktpreis für Rohöl herangezogen wird. 
ausschließlich auf der Grundlage der in einer Tonne Rohöl enthaltenen Mienge SKE berechnet und bei der Ermittlung von Abgaben an die Ölförderstaaten zugrundegelegt wird. Die Höhe dieses Preises schwankt um Pi. Und zum anderen der Marktpre is

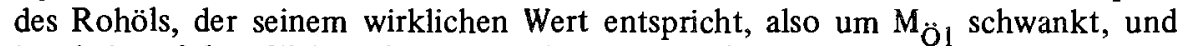
letztlich auf dem Weltmarkt auch realisiert wird, der also erheblich höher liegt als der fingierte ölpreis. Die letzten Rohölpreiserhöhungen seitens der Ölförderstaaten trafen ausschließlich die fingierten ölpreise und hatten nicht den geringsten Einfluß auf den wirklichen Marktpreis des Rohöls. Doch sollen im folgenden die oben aufgestellten Thesen näher begründet werden.

Legen wir die bekanntgegebenen Rohölpreise vom 1.10.1973 und 15. 10. 1973 am Persischen Golf zugrunde, wonach der Rohölpreis also pro Barrel (7,33 Barrel = 1 Tonne) erst 3,011 und dann 5,119 Dollar betrug (vgl. dazu Abb. 2), so ergibt sich bei dem damaligen Wechselkurs von 1 Dollar $=\mathrm{ca} .2,50 \mathrm{DM}$ am Persischen Golf am 1. 10. 1973 ein Rohölpreis von ca. 55 DM pro Tonne und am 16.10. desselben Jahres ein Preis von ca. 94 DM pro Tonne. Setzen wir diesem Preis noch die Frachtkosten hinzu, die laut offiziellen Angaben bis zum deutschen Verbrauchermarkt durchschnittlich $30 \mathrm{DM}$ pro Tonne betragen (vgl. dazu Abb. 4), so betrug der Rohölpreis trotz mehrfacher Erhöhung noch am 16. 10. 1973 vorigen Jahres auf dem deutschen Markt 124 DM pro Tonne.

Berücksichtigen wir nun, daß der Energiegehalt des Rohöls gegenüber dem der Steinkohle, bezogen auf die Tonne, in einem Verhältnis von 10 000:7 000 SKE bzw. 1,42: 1 SKE steht und daß der Produktionspreis Pi für die deutsche Steinkohle etwa 80-90 DM pro Tonne und 115-129 DM, auf 10000 SKE umgerechnet, beträgt, so sehen wir erstens, daß der Rohölpreis tatsächlich durch den Produktionspreis der in der Steinkohle enthaltenen Energiemenge reguliert wird (25), und wir sehen ferner, daß der bislang als Berechnungsgrundlage für die Abgaben an die ölförderstaaten zugrundegelegte Ölpreis lediglich den Preis für die darin enthaltene Energiemenge einschließt, die oben abgeleiteten Komponenten $\mathrm{Z}$ und $\mathrm{N}$ nicht beinhaltet, daher also erheblich unter dem wirklichen Rohölmarktwert liegt (26).

25) Die Tatsache, daß der Rohölpreis noch am 1. 10. 1973 auf dem deutschen Markt lediglich 55 DM plus Frachtkosten, also 85 DM pro Tonne, betragen hat, widerspricht dem Preiszusammenhang der Steinkohle und des Rohöls als Energieträger keineswegs, da der Preis von 85 DM pro Tonne Rohöl nur die Folge von permanenten, diesem Datum vorangegangenen Dollarabwertungen war. Mit der ersten erheblichen Preiserhöhung im Oktober von 3,011 auf 5,119 Dollar pro Barrel haben die Ölförderstaaten erst die negativen Folgen der Dollarabwertungen aufheben können. erst die negativen Folgen der Dollarabwertungen aufheben können.

26) Daß bisher die oben genannten Komponenten bei der Berechnung von Abgaben an die ÖIförderstaaten nicht berücksichtigt wurden, geht auch aus der Ankündigung des Schahs von Persien im Anschluß an das OPEC-Treffen im Dezember 1973 hervor, bei künftigen Preiskalkulationen diese Faktoren in Rechnung zu stellen. In einer Meldung der Frankfurter Rundschau vom 24.12.1973 heißt es dazu: „Nach den Worten des Schahs werden die Ölländer des Persischen Golfs künftig ihrer Preiskalkulation die Gestehungskosten alternativer Energiequellen, wie sie bei der Aufbereitung von Ölschiefer, bei der Verflüssigung von Kohle und bei der Erzeugung von Atomenergie entstünden, sowie den Mehrwert des Öls zugrundelegen, der sich aus seiner leichten Transportierbarkeit, seiner Umweltfreundlichkeit und aus den bei der Raffination anfallenden vielen wertvolen Nebenprodukten ergebe." Unabhängig davon, wie ernst auch diese Ankündigung ist, bringt sie doch zu- 
Es ist bekannt, daß die Ölförderstaaten dafür, daß sie die Rohölquellen an die ölkonzerne verpachten, einen prozentualen Anteil des von den Ölkonzernen realisierten Surplusprofits als Pachtgebühr erhalten. Es ist von daher also klar, daß die multinationalen ölkonzerne - und, wie wir sehen werden, auch die kapitalistischen Hauptverbraucherstaaten insgesamt - ein besonders großes Interesse daran haben, daß der als Berechnungsgrundlage für die Pachtgebühr an die Ölförderstaaten zugrundegelegte Rohölpreis so weit wie möglich unter den wirklichen Marktwert für Rohöl herabgedrückt wird. Würde nämlich nicht der fingierte Rohölpreis, sondern der wirkliche, dem Rohölmarktwert entsprechende Marktpreis für die Berechnung der Pachtgebühr zugrundegelegt werden, so wäre der Anteil der Ölförderstaaten am wirklich realisierten Surplusprofit um ein vielfaches höher als diesem tatsächlich gezahlt wird.

Nun sind aber in diesem Zusammenhang zwei wesentliche Fragen zu beantworten, nämlich wie hoch der wirkliche Marktwert für Rohöl ist und von wem die Masse des bei der Produktion von Rohöl auf dem Energiesektor wirklich anfallen. den Surplusprofits ange eignet wird.

Es ist sehr schwer, die Höhe des wirklichen Marktwertes für Rohöl zu bestimmen, weil sowohl die wirklichen Verarbeitungskosten des Rohöls zu Endprodukten wie der Erlös für die Ölnebenprodukte zu den Betriebsgeheimnissen der Mineralölkonzerne gehören und dies auch sicherlich aus ,wohl verständlichen Gründen“. Wir wollen aber versuchen, an Hand der spärlichen Angaben über die Preisstruktur des Mineralöls eines Ölkonzerns in der BRD, die wir in folgender graphischer Darstellung wiedergeben, die oben gestellten Fragen annähernd zu beantworten (27).

Aufgrund dieses Beispiels der Preisstruktur des Mineralöls im Jahre 1971 auf dem deutschen Energiemarkt be trägt der Konzernpreis für Mineralöl, allerdings nach der Verarbeitung zu Endprodukten, 135 DM pro Tonne. Von diesem Betrag die unbekannte Größe der Verarbeitungskosten abgezogen, ergibt sich der Rohölpreis der u. E. dem individuellen Produktionspreis der Steinkohle, in SKE umgerechnet (also der Kategorie Pi), entsprechen muß. Von dem Rohölpreis auf dem deutschen Markt die Frachtkosten abgezogen, ergibt sich der Rohölpreis, der für die Berechnung von Abgaben an die Ölförderstaaten zugrundegelegt wird.

Es fragt sich nun, was eigentlich mit den oben abgeleiteten Komponenten $\mathbb{Z}$ und $\mathbb{N}$ des wirklichen Marktwertes für Rohöl passiert. Denn, daß diese Komponen. ten bei der Berechnung der Abgaben an Ölförderstaaten überhaupt nicht berücksich-

nächst das eindeutige Zugeständnis der herrschenden Klasse in den Staaten am Persischen Golf zum Ausdruck, bisher auf einen erheblichen Teil des Werts ihrer nationalen Rohstoffe zugunsten des Imperialismus freiwillig verzichtet zu haben.

27) Die Angaben dieser graphischen Darstellung haben wir aus der Zeitschrift: Erdöl und Kohle ..., a. a. O., S. 717 übernommen. Hierzu ist folgendes zu bemerken:

1. Der Einfachheit halber haben wir den Betrag von -5 DM als Ergebnis der Gewinn- und Verlustrechnung nicht berücksichtigt.

2. Im Original sind die Gewinne der ölkonzerne unter ,Kosten für Verarbeitung + Transport + Lagerung + Vertrieb + Verwaltung" getarnt aufgeführt. Wir haben oben den entsprechenden Betrag von $56 \mathrm{DM} / \mathrm{t}$ Öl als "Gewinne der Ölkonzerne + Verarbeitungskosten" umschrieben.

3. Beim Verkaufspreis für Mineralöl von $265 \mathrm{DM} / \mathrm{t}$ handelt es sich um den durchschnittlichen Preis von Endprodukten. 


\section{$265 \mathrm{DM} / \mathrm{t}$}

Marktpreis

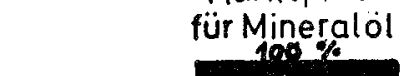
meralol

Förderkosten $3,1 \%$

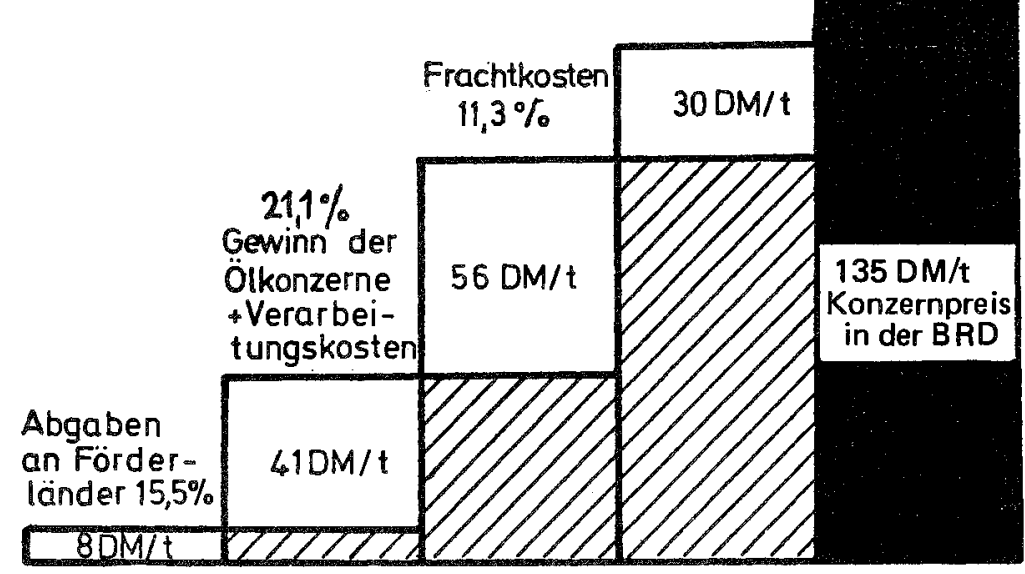

tigt werden, heißt noch lange nicht, daß sie auf dem Weltmarkt auch nicht realisiert werden.

Eine erste Antwort darauf kann erteilt werden, wenn man vor Augen führt, daß die Tonne importiertes Mineralöl im Jahre 1971 mit einem Steuersatz von nahezu $100 \%$ des Ölkonzernpreises belastet wird. Wie kommt denn der Staat zu dieser ungewöhnlich hohen Besteuerung, und ist gerade dieser Steuersatz eine willkürliche Größe? U.E. ist der Betrag von $130 \mathrm{DM} / \mathrm{t}$ ein unmittelbarer Bestandteil des wirklichen Marktwertes für Rohöl, der auf dem Wege der Besteuerung des importierten Mineralöls statt von den ölförderstaaten, von den kapitalistischen ölverbraucherstaaten angeeignet wird. Für diese These sprechen mindestens zwei Gründe:

1. Würde man statt des importierten öls die nationalen Hauptenergiequellen, z. B. die Steinkohle, anwenden, so würden die Preise für Endprodukte wahrscheinlich auch ohne Steuern im Durchschnitt genau so hoch liegen wie die 
gegenwärtigen Preise für die Endprodukte aus importiertem Mineralöl trotz so hoher Steuern. Demnach dürften sich die Mineralölsteuern mit der oben abgeleiteten Kategorie $\mathbf{Z}$ decken.

2. Obgleich die kapitalistischen Staaten unterschiedliche Steuerpolitik betreiben, ist es sicherlich nicht zufällig, daß sie alle das importierte Mineralöl ähnlich hoch belasten wie $\mathrm{z}$. B. in der BRD.

Aber auch selbst der die Steuern beinhaltende Marktpreis für Rohöl dürfte unter dessen wirklichem Marktwert liegen. Denn darin ist der Wert für die hochwertigen Ölnebenprodukte, also die Komponente $\mathrm{N}$ noch in keiner Weise berücksichtigt. Es ist auch unwahrscheinlich, daß die Mineralölsteuern den Wert der Ölnebenprodukte in irgendeiner Weise berücksichtigen. Diese Komponente kann nur aufgrund der Erlöse ermittelt werden, die die multinationalen Konzerne für den Verkauf der Ölnebenprodukte an die chemische Industrie erhalten. Daß der wirkliche Marktwert für Mineralöl höher ist als dessen Marktpreis, zeigt auch die Tatsache, daß seit 1971 z. B. sowohl der Berechnungspreis für die Abgabe an Ölförderstaaten (real um das Doppelte), wie auch die Mineralölsteuern wesentlich gestiegen sind (vgl. dazu $\mathrm{Abb}$. 5), ohne daß das importierte Rohöl gegenüber anderen Energieträgern an Konkurrenzfähigkeit verloren hätte. In der folgenden Abb. 5 (27a) ist die neue durchschnittliche Preisstruktur für die Erdölprodukte für das Jahr 1973 wiedergegeben. Diese Angaben sind zwar etwas detaillierter aufgeschlüsselt, weisen aber dieselben Probleme auf, wie wir sie bei den Angaben in der Abb. 4 feststellten. Das Neue und zugleich Interessante dabei ist, daß nun selbst die offiziellen Stellen die inzwischen gestiegenen Steuern als einen echten Bestandteil des Marktpreises für Rohöl offen zugeben.

27a) Quelle: Der Spiegel vom 31. 12.1973

\section{FISKUS IM TANK}

Zusammensetzung des Durchschnittspreises für ErdölProdukte in den wichtigsten Konsumentenländern 1973 (in Prozent)

Produktionskosten (Rohöl)

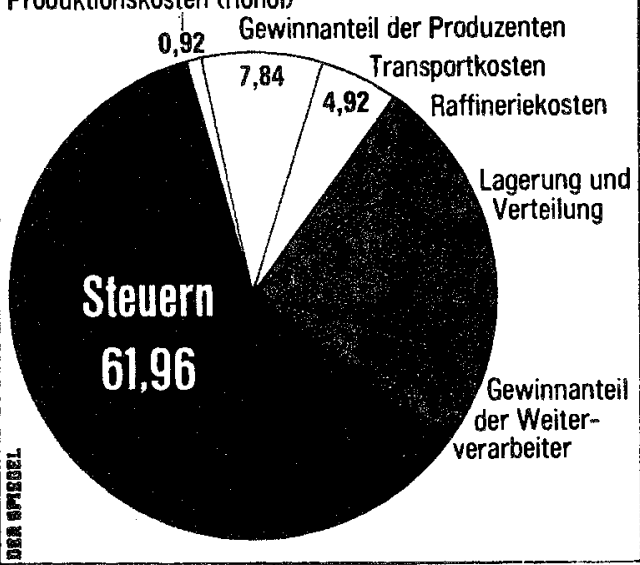


Es ist durchaus anzunehmen, daß der wirkliche Marktwert für Rohöl noch höher liegt als dessen Preis, der auch die erhöhten Steuern einschließt. Denn es ist allem Anschein nach auch für die nahe Zukunft nicht geplant, statt des importierten Rohöls Steinkohle oder andere Energiequellen in erheblichem Umfang zur Energiegewinnung anzuwenden, obgleich weitere Preiserhöhungen seitens der Ölförderstaaten nicht ausgeschlossen werden.

Der wirkliche Marktwert für Rohöl ist dann erreicht, wenn dessen offizieller Marktpreis so weit nach oben ansteigt, daß entweder Kapitale für die Gewinnung von Benzin, Heizol etc. auf die Anwendung von Steinkohle als Energierohstoff üBergehen, oder aber daß der Staat anfängt, die Mineralölsteuer zu senken.

Inzwischen hat sich aber auf dem Energiemarkt ein unbedeutender, aber in der Tendenz richtungweisender freier Ölmarkt durch die Ölversteigerung im Dezember 1973 in Teheran gebildet, wo das persische Öl, obgleich qualitativ sicherlich nicht das beste, zu einem Preis von 17 Dollar je Barrel verkauft wurde (28). Auf Tonne und DM bei einem Wechselkurs von 1 Dollar $=2,70 \mathrm{DM}$ umgerechnet, ist also die Tonne Rohöl zu einem Preis von ca. 336 DM am Persischen Golf verkauft worden. Wenn wir nun die Frachtkosten berücksichtigen, ist der Marktpreis für dieses Rohöl auf dem deutschen Markt ca. 366 DM pro Tonne.

Dieser Rohölpreis dürfte ziemlich nahe dem heute wirklichen Marktwert für Rohöl liegen, selbst wenn mann von einem Marktpreis von 265 DM pro Tonne im Jahre 1971 ausgeht (siehe Abb.4), also letzteren als den wirklichen Marktwert für Rohöl annimmt, und die Erhöhung des fingierten Rohölpreises sowie die inzwischen von $49 \%$ (Abb. 4) auf fast $62 \%$ (Abb. 5) gestiegenen Mineralölsteuern hinzuzählt.

Es stellt sich daher die wichtige Frage, weshalb die multinationalen Ölkonzerne, wo sie in den kapitalistischen Ölverbraucherstaaten als unmittelbare Verkäufer des Rohöls auftreten, das Rohöl nicht zu seinem wirklichen Marktwert bzw. Marktpreis verkaufen, weshalb sie also ,freiwillig“ einen Preis verlangen, auf den dann der kapitalistische Staat noch die gewaltig hohen Steuern zuschlagen kann? Was das Verhältnis der multinationalen Ölkonzerne zu den kapitalistischen Staaten betrifft, so ist es beiden Seiten gleichgültig, ob nun die Gewinne der Ölkonzerne entsprechend hoch besteuert werden oder aber dieselbe Steuermasse einem Ölverkaufspreis zugeschlagen wird, der um den Steuerbetrag niedriger ist als der wirliche Marktpreis. In beiden Fällen bleibt ein erheblicher Bestandteil des Rohölmarktwertes in den kapitalistischen Staaten.

Ganz anders wirkt sich aber die eine oder die andere Art von Besteuerung aus,

28) Der Spiegel vom 7. 1. 1974, S. 78, schreibt dazu: „Doch für 17 Dollar je Barrel gingen - obwohl der offizielle Verkaufspreis zu jener Zeit noch bei 3,65 Dollar lag (heute: rund 7 Dollar) - Mitte Dezember von den Persern versteigerte Spitzenmengen an Rohöl weg. Auf solch einer Preisbasis wird nahezu jede Art der Ölproduktion in die Bereiche der Rentabilität geschoben." Die Verfasser der Artikelserie im Spiegel, und nicht nur diese, übersehen aber, daß es sich bei dem, ,offiziellen Verkaufspreis" um den fingierten Preis für die Berechnung der Förderabgaben handelt. Der wirkliche Marktpreis für Rohöl, der auch auf dem Weltmarkt schon längst realisiert wird, bewegt sich um 17 Dollar je Barrel, und wahrscheinlich darüber, ohne daß aber irgend eine andere Art von Energieträgern bisher mit öl konkurrieren konnte. 
wenn man vom Verhältnis der multinationalen Konzerne und kapitalistischen Staaten einerseits $\mathrm{zu}$ den Ölförderstaaten andererseits ausgeht. Würden nämlich die Ölkonzerne das Rohöl auf den freien Märkten und zu dessen wirklichem Marktwert verkaufen, so erhöitte sich automatisch die absolute Höhe des Gewinnanteils der Ölförderstaaten, da der Gewinnanteil der Förderstaaten prozentual und vertraglich festgesetzt ist, so würde also dementsprechend der Teil des Rohölwerts sinken, der insgesamt in die kapitalistischen Staaten fließt. Der Anteil der Ölförderstaaten bleibt aber auf ein Minimum beschränkt, wenn die Ölkonzerne das Rohöl nicht auf den freien Energiemärkten verkaufen, dank ihrer Monopolstellung in der Sphäre den Rohölpreis auf einen wesentlich niedrigeren Preis festsetzen, der ungefähr dem Preis für dieselbe Menge SKE Steinkohle entspricht, und wenn die kapitalistischen Staaten, anstatt die hohen Gewinne der ölkonzerne entsprechend hoch zu besteuern, gewaltige Steuermassen dem fingierten Rohölpreis zuschlagen.

Dieser ungeheure Betrug an den ölförderstaaten funktioniert allerdings nur dann, wenn die multinationalen Ölkonzerne mit den kapitalistischen Ölverbraucherstaaten in allen wesentlichen Fragen der ölpreisstruktur eng zusammenarbeiten und die notwendigen Vereinbarungen treffen. Es gibt auch keinen Grund, an einer solchen urmittelbaren Liiertheit der Ölkonzerne mit den kapitalistischen Staaten zu zweifeln, wo es doch darum geht, einen höchstmöglichen Anteil des Rohölwertes von den ölförderländern in die Zentren der Kapitalakkumulation in der kapitalistischen Weltwirtschaft zu transferieren.

Die obige These kann auch durch die Tatsache unterstützt werden, daß in den kapitalistischen Ölverbraucherstaaten nur das importierte Rohöl so hoch besteuert wird, der Steuerbetrag auf das nationale Rohöl aber einen kleinen Bruchteil der Steuern auf importiertes Rohöl ausmacht (in der BRD beträgt der Steuersatz des nationalen Rohöls ca. $5 \%$ ). Und wenn in den USA die Rohölprodukte vergleichsweise billiger sind als in Europa und die Mineralölsteuer kaum ins Gewicht fällt, dann nur deshalb, weil die USA über $90 \%$ des Rohölbedarfs aus nationalen Quellen bestreiten, daher also eine künstliche Spaltung des Rohölpreises, wie es im Falle der anderen Staaten praktiziert wird, überflüssig ist.

Wir haben oben gesehen, daß die multinationalen Konzerne und die kapitalistischen Staaten versuchen, auf zwei Ebenen dc Anteil der Ölförderstaaten am wirklichen Wert des Rohöls so gering wie möglich zu halten. Auf der einen Seite durch künstliche Spaltung des Rohölpreises und durch die Zugrundelegung eines wesentlich niedrigeren Ölpreises für die Berechnung der Förderabgaben und auf der anderen Seite dadurch, daß, von diesem zu niedrig festgelegten Ölpreis ausgehend, selbst der Gewinnanteil der ölförderstaaten möglichst niedrig angesetzt wird. So kommt, daß, wie aus Abb. 5 hervorgeht, im Jahre 1973 die Ölförderstaaten insgesamt nur $7 \%$ des wirklichen Marktwertes ihres Rohöls erhielten, während fast $62 \%$ des Rohölwertes in Form von Steuern und etliche Prozente desselben von den multinationalen Konzernen angeeignet wurden.

Nach der erfolgreichen Tendenzwende im Verhältnis des Kapitals zum Grundeigentum versuchen die Ölförderstaaten ihren Anteil an dem erzielten Surplusprofit auf den obengenannten zwei Ebenen, aber in umgekehrter Richtung, zu steigern, nämlich zum einen, den künstlichen Rohölpreis so weit wie möglich dem wirklichen 
Marktwert des Rohöls anzunähern und zum anderen durch neue Verträge mit den multinationalen Konzernen die Förderabgaben zu erhöhen. $O b$ es diesen Staaten gelingt, den gesamten, ihnen gemäß der Logik der kapitalistischen Wertproduktion zufallenden Surplusprofit in Grundrente zu verwandeln und anzueignen, hängt da. von $a b, o b$ sie auch wirklich in der Lage sind, die Durchsetzung eines freien Energiemarktes in der kapitalistischen Weltwirtschaft zu erawingen. Alle Anzeichen sprechen aber dafür, daß die Regierungen kapitalistischer Staaten unter dem Deckmantel ,Solidarität" ihre Monopolposition gegenuber den Ölförderstaaten verstärken wollen, indem sie durch politische Vereinbarungen die aufgrund von entstandener Energieknappheit ausgelöste Konkurrenz untereinander wieder auszuschalten su. chen.

Wenn es auch den ölförderstaaten gelingen würde, ihren Anteil am gesamten Surplusprofit bei der Ölproduktion zu erhöhen, dürfte die Energiepolitik der kapitalistischen Staaten im wesentlichen unverändert bleiben. Ihre Politik besteht darin, die Rohölreserven der Welt, zumal sie in der Hauptsache in den unterentwickelten Gebieten liegen, auszuschöpfen, solange sie überhaupt einen Teil des dabei erzielten Surplusprofits, in welcher Form auch immer, abzweigen können. Die räuberische, also eindeutig imperialistische Energiepolitik geht aus den Prognosen offizieller Energieplaner hervor. Diese zeigen, wie aus der Abbildung 1 auch hervorgeht, daß bis zum Jahre 2000 die Produktion von Steinkohle absolut fast konstant bleibt, relativ aber um ein Vielfaches sinkt, obwohl fast $80 \%$ der bekannten Energiereserven der Welt in Form von Steinkohle vorkommen - deren bedeutender Anteil wiederum in Europa und in den USA liegen -, daß aber gleichzeitig bis zu diesem Datum die Produktion von Rohöl um $100 \%$ gesteigert werden soll, obwohl die bekannten Rohölreserven der Welt insgesamt nur $15 \%$ der derzeitigen Weltenergiereserven ausmachen.

\section{Resultate}

\subsection{Die Grenzen des kapitalistischen Wachstums}

Die obige Analyse der Wert- und Preisbildung sowie die der Aneignung des Surplusprofits in der Sphäre der Energieproduktion kann auf die gesamte extraktive Sphäre in der kapitalistischen Weltwirtschaft verallgemeinert werden, da auch in den übrigen Sphären der Rohstoffproduktion dem Wesen nach dieselben ökonomischen Mechanismen wirken wie in der Sphäre der Energieproduktion. Die extraktive Sphäre insgesamt betrachtet, wird erst klar, in welchem Umfang über lange Zeiträume das hohe Wachstum des Kapitalismus dadurch mitfinanziert wurde, daß für die Rohstoffe, soweit sie in den vorkapitalistischen Gesellschaften produziert wurden, effektiv ein Preis gezahlt wurde, der weit unter deren wirklichem Marktwert lag.

Doch diese Entwicklung war solange möglich, wie in den rohstoffreichen Gesellschaften der Welt ausschließlich vorkapitalistische Produktionsverhältnisse vorherrschten. Das Kapital, indem es sich in den vorkapitalistischen Gesellschaften als herrschendes Verhältnis durchsetzt, verwandelt das vorkapitalistische Grundei- 
gentum in seinen Gegensatz und reproduziert damit - wie alle anderen seiner Widerspriche - auch diesen Widerspruch seibst. Dem Wachstum der kapitalistischen Produktion in Zentren der Kapitalakkumulation werden nun erstens dadurch Grenzen gesetzt, daß die Konkurenz der Kapitale, die gïnstigere Naturbasis in den unterentwickelten Ländern für die Rohstoffproduktion anzuwenden, eingeschränkt wird, und zweitens dadurch, daß die Staaten der rohstoffreichen Gesellschaften beginnen, den Preis für ihre Rohstoffe zu fördern, der deren wirklichem Wert auch entspricht.

Die Tendenzwende in den Beziehungen zwischen den multinationalen Ölkonzernen und den Ölförderstaaten darf nur als der Beginn der Veränderung des Verhälmisses des Kapitals zum Grundeigentum äberhaupt angesehen werden. Erst die Durchsetzung dieses neuen Verhältnisses wird die neuen kapitalistischen Widersprüche in ihrem wirklichen Ausmał zum Tragen bringen.

Die Äußerungen der bürgerlichen Politiker lassen darauf schließen, daß sie selbst auch eine solche Entwicklung vermuten. Die Reaktion der imperialistischen Staaten auf diese mögliche Entwicklung kann man auf zwei Ebenen verfolgen.

A uf der einen Seite versuchen die kapitalistischen Staaten durch die erneute Verstärkung ihrer monopolistischen Machtposition gegenüber den rohstoffproduzierenden Ländern, nachdem sie offensichtlich von einer Tendenzwende ihrer Beziehungen zu jenen überrascht worden waren, die begonnene Veränderung des Kapitalverhältnisses zum Grundeigentum möglichst zu verschieben bzw. diese nicht voll wirksam werden zu lassen, wobei die Gewaltanwendung hierzu ganz offen als die letzte Möglichkeit nicht ausgeschlossen wird.

Auf der anderen Seite ist die Reaktion des Kapitals auf propagandistischer Ebene zu verfolgen. Während die ,nüchterne“ Kapitalfraktion und ihre Massenmedien die Massen psychologisch auf künftige Krisen (,Null-Wachstum, Massenarbeitslosigkeit etc. als Folge der steigenden Rohstoffpreise ${ }^{66}$ ) vorbereiten, sind die reaktionären Massenmedien dabei, Sündenböcke für die künftige Krise des Kapitalismus aufzubauen. Für die beginnende Krise des Kapitalismus, die offenbar vom Energiesektor auszugehen scheint, werden ,gie Araber" verantwortlich gemacht. Diese reaktionären Massenmedien gehen sogar so weit, selbst die kapitalistische Währungskrise bei der Gelegenheit den ,Arabern" zur Last zu legen. Mit dieser besonderen Art von Verschleierung der wirklichen Zusammenhänge gehen zahlreiche Zeitungen, Zeitschriften und Fernsehkommentatoren keineswegs sparsam um. Es wird der Eindruck erweckt, als würden die „ölscheichs", nachdem sie ,die kapitalistischen Staaten sogar durch Erpressung so schamlos ausbeuten", mit ihren Währungsreserven auch noch das kapitalistische Währungssystem zerstören, indem sie die Erzielung von Spekulationsgewinnen anstreben. Dieser ungeheuren Lüge werden zahlreiche Kommentare und Leitartikel gewidmet, wohingegen die Wahrheit nur am Rande Erwähnung findet:

\footnotetext{
„Die mit erhöhten Rohölpreisen verdienten Dollar-Milliarden der arabischen Förderländer können zum größten Teil nicht aus den Depots westlicher Banken abgezogen werden. Große Beträge der auf rund 50 Milliarden Dollar geschätz ten Reserven dienen zur Finanzierung bereits bestellter industrieller Ausrüstungsgüter. Det überwiegende Rest ist langfristig in Wertpapieren westlicher Industrieländer angelegt oder befindet sich auf Bankkonten in den USA, der Schweiz
} 
oder Großbritannien.

Zuverlässige Quellen bestätigen, daß beispielsweise die westlichen Devisenguthaben Iraks, das die Boykottpolitik nicht mitgemacht hat, den Wert der bestellten Importgüter derzeit um die Hälfte unterschreiten. Der Wert der von Saudi-Arabien bestellten Waren soll den gleichen Quellen zufolge den Wert der verfügbaren Devisenreserven des reichsten arabischen Ollandes um. etwa ein Drittel übersteigen.

So wird Frankreich in Kuwait und Abu Dhabi Industrieanlagen zur Verwertung von Erdölgasen im Wert von rund 120 Millionen Dollar bauen. Weiteres Geld benötigen diese beiden Länder, um für eine mit Frankreich betriebene Reederei eine Äthylen-Transportfotte zu bauen, die aus Schiffen von 30000 bis 40000 BRT bestehen soll. In Irak bewerben sich derzeir amerikanische und europäische Firmen um Aufträge für ähnliche Projekte " (29).

Diese offensichtlich auf „,zuverlässige Quellen“ gestützte Meldung beweist, wie gezielt die reaktionären Massenmedien Lügen verbreiten und die ganzen Miseren des Kapitalismus diesmal anstatt dem, internationalen Judentum ${ }^{66}$ dem ,internationa len Arabertum ${ }^{66}$ zuzuschieben suchen. Diese Rassenhaß schürende, dem Faschismus den besten Nährboden liefernde Propaganda wird um so gefăhrlicher, je langsamer und unentschiedener die Sozialisten ihr entgegenwirken.

\subsection{Die Verstaatlichung multinationaler Ölkonzerne - eine Illusion}

In den obigen Ausführungen wurde dargestellt, daß auf der Basis der kapitalistischen Wertproduktion der Wert und der Preis der in der extraktiven Sphäre produzierten Produkte nicht durch die wirklich in der Sphäre durchschnittlich angewandte Arbeitszeit, sondern durch die Arbeitszeit bestimmt wird, die für die Produktion desselben Produkts unter den ungünstigsten Bedingungen erforderlich ist.

An diesem Wertzusammenhang ändert sich auch nicht das geringste, wenn bestimmte Formen der Organisation durch andere ersetzt werden. Im aktuellen Zusammenhang ist also die Verstaatlichung der multinationalen ölkonzerne, wie sie von den Jungsozialisten und den linken Gewerkschaftskreisen gefordert wird, eine Illusion, würde man sich langfristig davon auf dem Energiemarkt eine Preisserkung und daher die Verbesserung des Lebensstandards versprechen. An dieser Situation würde sich unter Beibehaltung kapitalistischer Wertproduktion selbst dann nichts ändern, wenn die multinationalen Konzerne verstaatlicht, die ganze Welt sich in eine einzige Gesellschaft und die ganzen Staaten sich in einen einzigen Staat verwandeln würden. Unter den gegenwärtig gegebenen Bedingungen kommt den multinationalen Ölkonzernen, den kapitalistischen Staaten und den Ölförderstaaten insofern eine spezifische Bedeutung zu, als es um die Verteilung des auf der Basis der kapitalistischen Wertproduktion erzielten Surplusprofits geht.

Ganz anders stellt sich das Problem, wenn die kapitalistische Wertproduktion selbst aufgehoben ist. Was Marx im sechsten Abschnitt des dritten Bandes des „Kapital" in diesem Zusammenhang, bezogen auf eine kapitalistische Gesellschaft, schreibt, gilt heute für die gesamte kapitalistische Weltwirtschaft: 
„Es ist dies die Bestimmung duich den Marktwert, wie er sich auf Basis der kapitalistischen Produktionsweise vermittelst der Konkurrenz durchsetzt; diese erzeugt einen falschen sozialen Wert. Dies entspringt aus dem Gesetz des Marktwerts, dem die Bodenprodukte unterworfen werden. Die Bestimmung des Marktwerts der Produkte, also auch der Bodenprodukte, ist ein gesellschaftlicher Akt, wenrs auch ein gesellschaftlich unbewufl und unabsichtlich vollzogner, der mit Notwendigkeit auf dem Tauschwert des Produkts beruht, nicht auf dem Boden und den Differenzen seiner Fruchtbarkeit. Denkt man sich die kapitalistische Form der Gesellschaft aufgehoben und die Gesellschaft als bewußte und planmäßige Assoziation organisiert, so stellten die 10 qrs. eñ Quantum selbständiger Arbeitszeit vor, gleich dem, das in 240 sh. enthalten ist. Die Gesellschaft würde also dies Bodenprodukt nicht erkaufen zu dem $21 / 2$ fachen der wirklichen Arbeitszeit, die darin steckt; die Basis einer Klasse von Grundeigentümern fiele damit weg. Es würde dies ganz ebenso wirken wie eine Verwohlfeilerung des Produkts zu gleichem Betrag durch fremde Einfuhr. So richtig es daher ist zu sagen, daß - die jetzige Produktionsweise beibehalten, aber vorausgesetzt, daß die Differentialrente dem Staat zufiele - die Preise der Bodenprodukte bei sonst gleichbleibenden Umständen dieselben bleiben würden, so falsch ist es zu sagen, daß der Wert der Produkte derselbe bliebe bei Ersetzung der kapitalistischen Produktion durch Assoziation. Die Dieselbigkeit des Marktpreises für Waren derselben Art ist die Weise, worin sich der gesellschaftliche Charakter des Werts auf Basis der kapitalistischen Produktionsweise und uberhaupt der auf Warenaustausch zwischen einzelnen beruhenden Produktion durchsetzt. Was die Gesellschaft, als Konsument betrachtet, zuviel zahlt für die Bodenprodukte, was ein Minus der Realisierung ihrer Arbeitszeit in Bodenproduktion bildet, bildet jetzt das Plus für einen Teil der Gesellschaft, die Grundeigentümer" (30).

Also nicht die bloße Formveränderung, sondern die Aufhebung der kapitalistischen Wertproduktion ist es, die - um auf den aktuellen Zusammenhang Bezug zu nehmen - grundlegende Veränderungen in der Sphäre der Energieproduktion herbeifuihrt. Die ganze Frage der Verstaatlichung oder der Beibehaltung der multinationalen ölkonzerne reduziert sich also zwangsläufig darauf, ob nun der Staat oder die Konzerne besser in der Lage sind, den den Ölförderstaaten beraubten Teil des Surplusprofits effektiver in der kapitalistischen Wertproduktion einzusetzen.

Die illusionäre Forderung nach der Verstaatlichung der multinationalen Ölkonzerne, wenn sie von Sozialisten erhoben und propagiert wird, wird insofern sogar schädlich, als sie dem Proletariat als eine Alternative erscheint und sie es dadurch davon ablenkt, die wirklichen Zusammenhänge zu erkennen. Dem Proletariat geht es aber darum, die tiefgreifenden Zusammenhänge und Beziehungen des ihm gegenüber fremden gesellschaftlichen Verhältnisses zunächst zu erkennen, will es über sein Schicksal als gesellschaftliche Klasse endlich selbst bestimmen. In der Erforschung und Erkenntnis eben dieser Beziehungen sowie in der Vermittlung derselben innerhalb des Proletariats findet die sozialistische Intelligenz ihre wichtig. ste Aufgabe. 